\title{
Optimization Algorithms As Training Approach With Deep Learning Methods To Develop An Ultraviolet Index Forecasting Model
}

Abul Abrar Masrur Ahmed ( $\square$ abulabrarmasrur.ahmed@usq.edu.au )

University of Southern Queensland https://orcid.org/0000-0002-7941-3902

Mohammad Hafez Ahmed

West Virginia University

Sanjoy Kanti Saha

Norwegian University of Science and Technology: Norges teknisk-naturvitenskapelige universitet

Oli Ahmed

Leading University

Ambica Sutradhar

Leading University

\section{Research Article}

Keywords: Deep Learning, Hybrid Model, Solar Ultraviolet Index, Optimization Algorithms, Public Health

Posted Date: September 21st, 2021

DOl: https://doi.org/10.21203/rs.3.rs-886915/v1

License: (9) This work is licensed under a Creative Commons Attribution 4.0 International License.

Read Full License 
1 Optimization algorithms as training approach with deep learning methods to develop an ultraviolet index forecasting model

\section{A. A. Masrur Ahmed:}

Email: masrur@outlook.com.au

Mohammad Hafez Ahmed*: 
Abstract: The solar ultraviolet index (UVI) is a key public health indicator to mitigate the ultraviolet-exposure related diseases. However, in practice, the ultraviolet irradiance measurements are difficult and need expensive ground-based physical models and timeconsuming satellite-observed data. Furthermore, accurate short-term forecasting is crucial for making effective decisions on public health owing to UVI related diseases. To this end, this study aimed to develop and compare the performances of different hybridized deep learning models for forecasting the daily UVI index. The ultraviolet irradiance-related data were collected for Perth station of Western Australia. A hybrid-deep learning framework was formulated with a convolutional neural network and long short-term memory called CLSTM. The comprehensive dataset (i.e., satellite-derived Moderate Resolution Imaging Spectroradiometer, ground-based datasets from Scientific Information for Landowners, and synoptic-scale climate indices) were fed into the proposed network and optimized by four optimization techniques. The results demonstrated the excellent forecasting capability (i.e., low error and high efficiency) of the recommended hybrid CLSTM model compared to the counterpart benchmark models. Overall, this study showed that the proposed hybrid CLSTM model successfully apprehends the complex and non-linear relationships between predictor variables and the daily UVI. A complete ensemble empirical mode decomposition with adaptive noise (CEEMDAN)-CLSTM-based is appeared to be an accurate forecasting system capable of reacting quickly to measured conditions. Further, the genetic algorithm is found to be the most effective optimization technique. The study inference can considerably enhance real-time exposure advice for the public and help 
44 Keywords: Deep Learning; Hybrid Model; Solar Ultraviolet Index; Optimization Algorithms;

45 Public Health.

46

47 List of abbreviations:

\begin{tabular}{ll}
\hline ACO & Ant Colony Optimization \\
\hline ACF & Autocorrelation Function \\
\hline ANFIS & Adaptive Neuro-Fuzzy Inference System \\
\hline ANN & Artificial Neural Network \\
\hline AO & Arctic Oscillation \\
\hline ARPANSA & Australian Radiation Protection and Nuclear Safety Agency \\
\hline BCC & Basal Cell Carcinoma \\
\hline BOM & Bureau of Meteorology \\
\hline CEEMDAN & Complete Ensemble Empirical Mode Decomposition with Adaptive \\
& Noise \\
\hline CEEMDAN-CLSTM & Hybrid Model integrating the CEEMDAN and CNN algorithm with \\
\hline CEEMDAN-CGRU & LSTM \\
\hline CEEMDAN-GRU & Hybrid Model integrating the CEEMDAN and CNN algorithm with \\
\hline CNN-LSTM (or CLSTM) & Hybrid model integrating the CEEMDAN algorithm with GRU \\
\hline CNN-GRU (or CGRU) & Hybrid model integrating the CNN algorithm with GRU \\
\hline CEEMDAN & Complete ensemble empirical mode decomposition with Adaptive \\
\hline CEEMDAN-DT & Noise \\
\hline CEEMDAN-MLP & Hybrid model integrating the CEEMDAN algorithm with DT \\
\hline CEEMDAN-SVR & Hybrid model integrating the CEEMDAN algorithm with MLP \\
\hline CNN & Hybrid model integrating the CEEMDAN algorithm with SVR \\
\hline COVID-19 & Convolutional Neural Network \\
\hline CCF & Coronavirus disease 2019 \\
\hline EEMD & Cross-Correlation Function \\
\hline EMD & Ensemble empirical mode decomposition \\
\hline DEV & Empirical Mode Decomposition \\
\hline DL & differential evolution \\
\hline DT & Deep Learning \\
\hline DWT & Decision Tree \\
\hline ECDF & Discrete wavelet Transformation \\
\hline ELM & Empirical Cumulative Distribution Function \\
\hline & Extreme Learning Machine \\
\hline & \\
\hline
\end{tabular}




\begin{tabular}{ll}
\hline EMI & El-Nino southern oscillation Modoki indices \\
\hline ENSO & El Niño Southern Oscillation \\
\hline FE & Forecasting Error \\
\hline GA & Genetic Algorithm \\
\hline GB & Giga Bite \\
\hline GIOVANNI & $\begin{array}{l}\text { Geospatial Online Interactive Visualization and Analysis } \\
\text { Infrastructure }\end{array}$ \\
\hline GRU & Gated Recurrent Unit \\
\hline GLDAS & Global Land Data Assimilation System \\
\hline GSFC & Goddard Space Flight Centre \\
\hline IMF & Intrinsic mode functions \\
\hline LM & Legates-McCabe's Index \\
\hline LSTM & Long- short term memory \\
\hline MAE & Mean Absolute Error \\
\hline MAPE & Mean Absolute Percentage Error \\
\hline MARS & Multivariate Adaptive Regression Splines \\
\hline MDB & Murray-Darling Basin \\
\hline MJO & Madden-Julian Oscillation \\
\hline ML & Machine Learning \\
\hline MLP & Multi-Layer Perceptron \\
\hline MODWT & Maximum Overlap Discrete Wavelet Transformation \\
\hline MODIS & Moderate Resolution Imaging Spectroradiometer \\
\hline MRA & Multi-resolution Analysis \\
\hline MSE & Mean Squared Error \\
\hline NAO & North Atlantic Oscillation \\
\hline NASA & National Aeronautics and Space Administration \\
\hline NCEP & National Centers for Environmental Prediction \\
\hline NO & Nitrogen Oxide \\
\hline NOAA & National Oceanic and Atmospheric Administration \\
\hline NMSC & Non-melanoma Skin Cancer \\
\hline NSE & Nash-Sutcliffe Efficiency \\
\hline PACF & partial autocorrelation function \\
\hline PDO & Pacific Decadal Oscillation \\
\hline PNA & Pacific North American \\
\hline PSO & Particle Swarm Optimization \\
\hline RMM & Correlation Coefficient \\
\hline BRF & Genetic Algorithm \\
\hline
\end{tabular}




\begin{tabular}{ll}
\hline RMSE & Root-Mean-Square-Error \\
\hline RNN & Recurrent Neural Network \\
\hline RRMSE & Relative Root-Mean-Square Error \\
\hline SAM & Southern Annular Mode \\
\hline SARS-CoV-2 & Severe Acute Respiratory Syndrome Coronavirus 2 \\
\hline SCC & Squamous Cell Carcinoma \\
\hline SILO & Scientific Information for Landowners \\
\hline SOI & Southern Oscillation Index \\
\hline SST & Sea Surface Temperature \\
\hline SVR & Support Vector Regression \\
\hline US & United States \\
\hline UV & Ultraviolet \\
\hline UVI & Ultraviolet Index \\
\hline WHO & World Health Organization \\
\hline WI & Willmott's Index of Agreement
\end{tabular}

\section{Introduction}

Solar ultraviolet (UV) radiation is an essential component for the sustenance of life on

51 Earth (Norval et al., 2007). The UV irradiance consists of a small fraction (e.g., 5-7\%) of the total

52 radiation and produces numerous beneficial effects on human health. It has been in use since the

53 ancient time for improving the body's immune system, such as strengthening of bones and muscles

54 (Juzeniene and Moan, 2012) as well as in treating various hard-to-treat skin diseases such as atopic

55 dermatitis, psoriasis, phototherapy of localized scleroderma (Furuhashi et al., 2020; Kroft et al.,

56 2008), and vitiligo (Roshan et al., 2020). UV-stimulated tanning has been proved as a positive

57 mood changing and relaxing effect for many (Sivamani et al., 2009). Further, UV-induced nitrogen

58 oxide (NO) plays a vital role in reducing human blood pressure (Juzeniene and Moan, 2012;

59 Opländer Christian et al., 2009).

60 UV light has also been widely used as an effective disinfectant in the food and water

61 industry to inactivate disease-producing microorganisms (Gray, 2014). Because of no harmful by-

62 products generation and its effectiveness against protozoa contamination, the use of UV light as a 
63 drinking water disinfectant has achieved an increased acceptance (Timmermann et al., 2015). To 64 date, most of the UV-installed public water supplies are in Europe. In the United States (US), its 65 application is mainly limited to groundwater treatment (Chen et al., 2006). However, its use is 66 expected to increase in the future for the disinfection of different wastewater systems. Developing 67 countries worldwide find it useful as it offers a simple, low-cost, and effective disinfection 68 technique in water treatment compared to the traditional chlorination method (Mäusezahl et al., 69 2009; Pooi and Ng, 2018).

The application of UV light has also shown potency in fighting airborne-mediated diseases

71 for a long time (Hollaender et al., 1944; Wells and Fair, 1935). For instance, a recent study

72 demonstrated that a small dose (i.e., $2 \mathrm{~mJ} / \mathrm{cm}^{2}$ of $222-\mathrm{nm}$ ) of UV-C light can efficiently inactivate 73 aerosolized H1N1 influenza viruses (Welch et al., 2018). The far UV-C light can also be applied

74 in sterilizing surgical equipment. Recently, the use of the UV-C light as the surface disinfectant 75 has been significantly increased to combat the global pandemic (COVID-19) caused by 76 coronavirus SARS-CoV2. A recent study also highlighted the efficacy of UV light application on 77 the disinfection of COVID-19 surface contamination (Heilingloh et al., 2020).

However, the research on UV radiation has also been a serious concern over the years due

79 to its dichotomy nature. UV irradiance can also have detrimental biological effects on human

80 health, such as skin cancer and eye disease (Lucas et al., 2008; Turner et al., 2017). Chronic 81 exposure to UV light has been reported as a major risk factor responsible for melanoma and non82 melanoma cancers (Saraiya et al., 2004; Sivamani et al., 2009) and associated with 50-90\% of 83 these diseases. In a recent study, the highest global incidence rates of melanoma were observed in 84 the Australasia region compared to other North American and European parts (Karimkhani et al., 85 2017). Therefore, it is crucial to provide correct information about the intensity of UV irradiance 
86 to the people at risk to protect their health. This information would also be helpful for working

87 people in different sectors (e.g., agriculture, medical sector, water management, etc.).

The World Health Organization (WHO) formulated the global UV index (UVI) as a numerical public health indicator to convey the associated risk when exposed to UV radiation

90 (Fernández-Delgado et al., 2014; WHO, 2002). However, the UV irradiance estimation in practice

91 requires ground-based physical models (Raksasat et al., 2021) and satellite-derived observing

92 systems with advanced technical expertise (Kazantzidis et al., 2015). The installation of required

93 equipment (i.e., spectroradiometers, radiometers, and sky images) is expensive (Deo et al., 2017)

94 and difficult for remote regions, primarily mountainous areas. Furthermore, the solar irradiance is

95 also highly impacted by many hydro-climatic factors, e.g., clouds and aerosol (Li et al., 2018;

96 Staiger et al., 2008) and ozone (Baumgaertner et al., 2011; Tartaglione et al., 2020) that can insert

97 considerable uncertainties into the available process-based and empirical models (detail also given

98 in method Section). Therefore, the analysis of sky images may also require extensive bias

99 corrections, i.e., cloud modification (Krzyścin et al., 2015; Sudhibrabha et al., 2006), which creates

100 further technical as well as a computational burden. An application of data-driven models can be 101 useful to minimize these formidable challenges. Specifically, the non-linearity into data matrix can 102 easily be handled using data-driven models that a traditional process-based and/ semi-process103 based model fails. Further, the data-driven models are easy to implement, do not demand high 104 process-based cognitions (Qing and Niu, 2018; Wang et al., 2018), and are computationally less 105 burdensome.

As an alternative to conventional process-based and empirical models, applying different

107 machine learning (ML) algorithms as data-driven models has proven tremendously successful 108 because of the powerful computational efficiency. With technological advancement, 
109 computational efficiency has been significantly increased, and researchers have developed many

110 ML tools. Artificial neural networks (ANNs) are the most common and extensively employed in

111 solar energy applications (Yadav and Chandel, 2014). However, many studies, such as the multiple

112 layer perceptron (MLP) neural networks (Alados et al., 2007; Alfadda et al., 2018), support vector

113 regression (SVR) (Fan et al., 2020; Kaba et al., 2017), decision tree (Jiménez-Pérez and Mora-

114 López, 2016), and random forest (Fouilloy et al., 2018) have also been extensively applied in

115 estimating the UV erythemal irradiance. The multivariate adaptive regression splines (MARS) and

116 M5 algorithms were applied in a separate study for forecasting solar radiation (Srivastava et al.,

117 2019). Further, the deep learning network such as the convolutional neural network (CNN)

118 (Szenicer et al., 2019) and the long short-term memory (LSTM) (Huang et al., 2020; Qing and

119 Niu, 2018; Raksasat et al., 2021) are recent additions in this domain.

However, the UVI indicator is more explicit to common people compared to UV irradiance

121 values. Further, only a few data-driven models have been applied for UVI forecasting. For

122 example, an ANN was used in modeling UVI on a global scale (Latosińska et al., 2015). An

123 extreme learning method (ELM) was applied in forecasting UVI in the Australian context (Deo et

124 al., 2017). To date, there have not been many studies that used ML methods to forecast UVI. Albeit

125 the successful predictions of these standalone ML algorithms, they have architectural flaws and

126 predominantly suffer from overfitting efficiency (Ahmed and Lin, 2021). Therefore, the hybrid

127 deep learning models receive increased interest and are extremely useful in predictions with higher

128 efficiency than the standalone machine learning models. Hybrid models such as particle swarm

129 optimization (PSO)-ANN (Isah et al., 2017), wavelet-ANN (Zhang et al., 2019), genetic algorithm

130 (GA)-ANN (Antanasijević et al., 2014), Boruta random forest (BRF)-LSTM (Ahmed et al., 2021;

131 Ahmed et al., 2021b), ensemble empirical mode decomposition (EEMD) (Liu et al., 2015), 
132 adaptive neuro-fuzzy inference system (ANFIS)-ant colony optimization (ACO) (Pruthi and 133 Bhardwaj, 2021) and (ACO)-CNN-GRU (Ahmed et al., 2021b) have been applied across 134 disciplines and attained substantial tractions. However, a CNN-LSTM (i.e., CLSTM) hybrid model 135 can efficiently extract inherent features from the data matrix than other machine learning models 136 and has successfully predicted time series air quality and meteorological data (Pak et al., 2018).

137 The application of such a hybrid model for predicting sequence data, i.e., the UVI for consecutive 138 days, can be an effective tool with excellent predictive power. However, the forecasting of UVI 139 with a CLSTM hybrid machine learning model is yet to be explored and was a key motivation for 140 conducting this present study.

141 The conventional MRA, for instance, discrete wavelet transform (DWT), has been 142 implemented for a long time (Deo and Sahin, 2016; Deo et al., 2016; Nourani et al., 2014; Nourani 143 et al., 2009), but there appear to be disadvantages that preclude the full feature extraction, as well 144 as a unique destination of training features into a tested dataset. MODWT, as an advanced DWT, 145 can identify critical issues (Cornish et al., 2006; Prasad et al., 2017; Rathinasamy et al., 2014). In 146 this study, we employed a new model of EMD called complete ensemble empirical mode 147 decomposition with adaptive noise (CEEMDAN) (Prasad et al., 2018). in CEEMDAN-based 148 decomposition, Gaussian white noise with a unit variance is added consecutively at each stage to 149 reduce the forecasting procedure's complexity (Di et al., 2014). Previous studies used CEEMDAN 150 in predicting soil moisture (Ahmed et al., 2021a; Prasad et al., 2018; Prasad et al., 2019). However, 151 a previous version (i.e., EEMD) was used in forecasting streamflow (Seo and Kim, 2016) and 152 rainfall (Beltrán-Castro et al., 2013; Jiao et al., 2016; Ouyang et al., 2016). The only machine 153 learning algorithm used in the study is CLSTM, which has not been coupled with the EEMD or 154 CEEMDAN to produce a UVI forecast system. 
This study aims to apply a CLSTM hybrid machine learning model, which can exploit the

156 benefits of both convolutional layers (i.e., important feature extraction) and LSTM layers (i.e.,

157 storing sequence data for an extended period) and evaluate its ability to efficiently forecast the

158 UVI for the next day. The model was constructed and fed with hydro-climatic data in association

159 with UV irradiance in the Australian context. The model was optimized using ant colony

160 optimization, genetic algorithm, particle swarm optimization, and differential evolutional

161 algorithms. The model accuracy (i.e., efficiency and errors involved in UVI estimations) was

162 assessed with the conventional standalone data-driven models' (e.g., SVR, decision tree, MLP,

163 CNN, LSTM, gated recurrent unit (GRU), etc.) performance statistics. The inference obtained

164 from the modeling results was also discussed that could be tremendously useful in building expert

165 judgment to protect public health in the Australian region and beyond.

\section{2. Materials and Methods}

\section{$167 \quad 2.1$ Study area and UVI data}

The study assessed the solar ultraviolet index of Perth (Latitude: $-31.93^{\circ} \mathrm{E}$ and Longitude:

$\left.169115.10^{\circ} \mathrm{S}\right)$, Western Australia. The Australian Radiation Protection and Nuclear Safety Agency

170 (ARPANSA) provided the UVI data of Australia (ARPANSA, 2021). Figure 1 shows the monthly

171 UVI data, the location of Perth, and the assessed station. The figure shows that Perth has low to

172 extreme UV concentration over the year between 1979-2007. The Summer season (December to

173 February) had the most extreme UV concentration, whereas the Autumn (March to May) has

174 moderate to high, and Winter (June to August) demonstrates lower to moderate, and Spring

175 (September to November) has higher to extreme UVI value in Perth. 
Malignant melanoma rates in Western Australia are second only to those in Queensland,

177 Australia's most populated state (Slevin et al., 2000). Australia has the highest incidence of NMSC

178 (Non-melanoma skin cancer) globally (Anderiesz et al., 2006; Staples et al., 1998). Approximately

179 three-quarters of the cancer cases have basal cell carcinoma (BCC) and squamous cell carcinoma

180 (SCC) types. These are attributed to the fair-skinned population's high exposure to ambient solar

181 radiation (Boniol, 2016; McCarthy, 2004). As a result, Australia is seen as a world leader in public

182 health initiatives to prevent and detect skin cancer. Programs that have brought awareness of

183 prevention strategies and skin cancer diagnoses have data to show that people act on their

184 knowledge (Stanton et al., 2004). Many research showed that reducing sun security measures is

185 linked to reducing rates of BCC and SCC in younger groups. They might have received cancer

186 prevention messages as children (Staples et al., 2006). Considering the diversified concentration

187 of UVI concentration, this study considers Perth as an ideal study area.

\section{$188 \quad 2.2$ Datasets of predictor variables}

189 Three distinct data sources were used to collect the predictor variables in this analysis. The

190 Moderate Resolution Imaging Spectroradiometer (MODIS) satellite datasets are used to capturing

191 land surface status and flow parameters at regular temporal resolutions. These are supplemented

192 by ground-based Scientific Information for Landowners (SILO) repository meteorological data for

193 biophysical modeling and climate mode indices to help achieve Sea Surface Temperature (SST)

194 over Australia. Geospatial Online Interactive Visualization and Analysis Infrastructure

195 (GIOVANNI) is a geoscience data repository that provides a robust online visualization and 196 analysis platform for geoscience datasets. It collects data from over 2000 satellite variables (Chen 197 et al., 2010). The MODIS- aqua yielded 8 predictor variables for our study: a high-temporal 198 terrestrial modeling system consisting of a surface state and providing daily products with a high 
resolution (250 $\mathrm{m}$ at nadir). A list of predictors of the MODIS Satellite can be obtained from the

200 National Aeronautics and Space Administration (NASA) database (Giovanni, 2021).

The surface UVI is influenced by atmospheric attenuation of incident solar radiation (Deo et al., 2017). The angle subtended from the zenith $(\theta \mathrm{s})$ to the solar disc is another factor that affects the intensity of solar UV radiation (Allaart et al., 2004). The ultraviolet attenuation of clear-sky

204 solar radiation is dependent on ozone and atmospheric aerosol concentrations, along with cloud

205 cover (Deo et al., 2017). This implies that the measurements of biologically effective UV 206 wavelengths are affected by total column ozone concentration. Incident radiation at the Earth's 207 surface is reduced by aerosols such as dust, smoke, and vehicle exhausts (Downs et al., 2016; 208 Román et al., 2013). Moreover, Lee et al. (2009) found a significant correlation between UV solar 209 radiation and geopotential height. Considering the direct influence of the predictors over ultraviolet 210 radiation and UV index, this study collected Ozone total column, aerosol optical depth (550nm 211 and $342.5 \mathrm{~nm}$ ), geopotential height, cloud fraction, and combined cloud optical thickness data from 212 the Geospatial Online Interactive Visualization and Analysis Infrastructure (GIOVANNI) 213 repository.

214 Therefore, meteorological predictor variables (i.e., temperature, u- and v-winds) were 215 significant while modeling UVI (Lee et al., 2009). Moreover, the cloud amount and diurnal 216 temperature range have a strong positive correlation, while rainfall and cloud amount show a 217 strong negative correlation (Jovanovic et al., 2011). Although overall cloud patterns agree with 218 rainfall patterns across Australia, the higher-quality cloud network is too coarse to represent 219 topographic influences accurately. Changes in the amount of cloud cover caused by climate change 220 can result in long-term changes in maximum and minimum temperature. Owing to the relations of 221 hydro-meteorological variables with UVI and their interconnections with cloud cover, the study 
222 selected nine meteorological variables from the Scientific Knowledge for Land-Owners (SILO)

223 database to expand the pool of predictor variables, allowing for more practical application and

224 model efficiency. SILO data are managed by Queensland's Department of Environment and

225 Research and can be obtained from https://www.longpaddock.qld.gov.au/silo.

226 Aerosol-rainfall relationships are also likely to be artifacts of cloud and cloud-clearing

227 procedures. During the Madden-Julian Oscillation (MJO) wet phase, the high cloud's value

228 increases, the cloud tops rise, and increased precipitation enhances wet deposition, which reduces

229 aerosol mass loading in the troposphere (Tian et al., 2008). The MJO (Lau and Waliser, 2011;

230 Madden and Julian, 1994, 1971) dominates the intra-seasonal variability in the tropical

231 atmosphere. A relatively slow-moving, large-scale oscillation in the deep tropical convection and

232 baroclinic winds exists in the warmer tropical waters in the Indian and western Pacific Oceans

233 (Hendon and Salby, 1994; Kiladis et al., 2001; Tian et al., 2008). The study used the Real-time

234 Multivariate MJO series 1 (RMM1) and 2 (RMM2) obtained from the Bureau of Meteorology,

235 Australia (BOM, 2020). Though RMM1 and RMM2 indicate an evolution of the MJO independent

236 of season, the coherent off-equatorial behavior is strongly seasonal (Wheeler and Hendon, 2004).

237 Pavlakis et al. $(2008,2007)$ studied the spatial and temporal variation of long surface wave and

238 short wave radiation. A high correlation was found between the longwave radiation anomaly and

239 the Niño3.4 index time series over the Niño3.4 region located in the central Pacific.

240 Moreover, Pinker et al. (2017) investigated the effect of El Niño and La Nina cycles on

241 surface radiative fluxes and the correlations between their anomalies and a variety of El Niño

242 indices. The maximum variance of anomalous incoming solar radiation is located just west of the

243 dateline. It coincides with anomalous SST (Sea surface temperature) gradient in the traditional

244 eastern Pacific El Niño Southern Oscillation (ENSO). However, we derive the Southern 
245 Oscillation Index highly correlated with solar irradiance and mean Northern Hemisphere 246 temperature fluctuations reconstructions (Yan et al., 2011). In North America and the North

247 Pacific, land and sea surface temperatures, precipitation, and storm tracks are determined mainly 248 by atmospheric variability associated with the Pacific North American (PNA) pattern. The modern 249 instrumental record indicates a recent trend towards a positive PNA phase, which has resulted in 250 increased warming and snowpack loss in northwest North America (Liu et al., 2017). This study 251 used fifteen climate mode indices to increase the diversity.

\section{$252 \quad$ 2.3 Standalone models}

\section{$253 \quad$ 2.3.1 Multiple layer perceptron (MLP)}

254 The MLP is a simple feedforward neural network with three layers and is commonly used as a 255 reference model for comparison in machine learning application research (Ahmed and Lin, 2021). 256 The three layers are the input layer, a hidden layer with n-nodes, and the output layer. The input 257 data are fed into the input layer, transformed into the hidden layer via a non-linear activation 258 function (i.e., a logistic function). The target output is estimated, Eq. (1).

$259 y=f\left(\sum w^{T} x+b\right)$

260 where $w=$ the vector of weights, $x_{i}=$ the vector of inputs, $b=$ the bias term; $f=$ the non-linear

261 sigmoidal activation function, i.e., $f(z)=\frac{1}{1+e^{-z}}$.

262 The computed output is then compared with the measured output, and the corresponding loss, i.e., 263 the mean squared error (MSE), is estimated. The model parameters (i.e., initial weights and bias) 264 are updated using a backpropagation method until the minimum MSE is obtained. The model is 265 trained for several iterations and tested for new data sets for prediction accuracy. 
267 The SVR is constructed based on the statistical learning theory. In SVR, a kernel trick is applied 268 that transfers the input features into the higher dimension to construct an optimal separating 269 hyperplane as follows (Ji et al., 2017):

$270 \quad f(x)=w \cdot \varphi(x)+b$

271 where $w$ is the weight vector, $b$ is the bias, and $\varphi(x)$ indicates the high-dimensional feature space.

272 The coefficients $w$ and $b$, which define the location of the hyperplane, can be estimated by 273 minimizing the following regularized risk function:

274 Minimize: $\frac{1}{2}\left\|w^{2}\right\|+C \sum_{1}^{n}\left(\varepsilon_{i}+\varepsilon_{i}^{*}\right)$

275 Subject to $y_{i}-w \cdot \varphi(x)-b \leq \varepsilon+\varepsilon_{i} ; w \cdot \varphi(x)+b-y_{i} \leq \varepsilon+\varepsilon_{i}^{*} ; \varepsilon_{i} \leq 0 ; \varepsilon_{i}^{*} \leq 0$

276 where $C$ is the regularization parameter, $\varepsilon_{i}$ and $\varepsilon_{i}^{*}$ are slack variables. The Eq. (7) can be solved in 277 a dual form using the Lagrangian multipliers as follows:

278 Maximize: $-\frac{1}{2} \sum_{i=1}^{n} \sum_{j=1}^{n}\left(a_{i}-a_{i}^{*}\right)\left(a_{j}-a_{j}^{*}\right) K\left(x_{i}, x_{j}\right)-\sum_{i=1}^{n}\left(a_{i}-a_{i}^{*}\right)+\sum_{1}^{i=n}\left(a_{i}-a_{i}^{*}\right) y_{i}$

280 Subject to $\sum_{i=1}^{n}\left(a_{i}-a_{i}^{*}\right)=0 ; a_{i}, a_{i}^{*} \in[0, C]$

281 Where, $K\left(x_{i}, x\right)$ is the non-linear kernel function. In this present study, we used a radial basis 282 function $(\mathrm{RBF})$ as the kernel, which is represented as follows:

$283 K\left(x_{i}, x\right)=\exp \left(\frac{-\left\|x-x_{i}\right\|^{2}}{2 \sigma^{2}}\right)$, where $\sigma$ is the bandwidth of the RBF.

\section{$284 \quad$ 2.3.3 Decision tree (DT)}

285 A decision tree is a predictive model used for both classification and regression analysis (Jiménez286 Pérez and Mora-López, 2016). As our data is continuous, we used it for the regression predictions. 287 It is a simple tree-like structure that uses the input observations (i.e., $\mathrm{x}_{1}, \mathrm{x}_{2}, \mathrm{x}_{3}, \ldots, \mathrm{x}_{\mathrm{n}}$ ) to predict 
the target output (i.e., Y). The tree contains many nodes, and at each node, a test to one of the

289 inputs (e.g., $\mathrm{x}_{1}$ ) is applied, and the outcome is estimated. The left/right sub-branch of the decision

290 tree is selected based on the estimated outcome. After a specific node, the prediction is made, and

291 the corresponding node is termed the leaf node. The prediction averages out all the training points

292 for the leaf node. The model is trained using all input variables and corresponding loss; the mean

293 squared error (MSE) is calculated to determine the best split of the data. The number of maximum

294 features is set as the total input features during the partition.

\subsubsection{Convolutional neural network (CNN)}

The CNN model was originally developed for document recognition (Lecun et al., 1998)

297 and used for predictions. Aside from the input and output layer, the CNN architecture has three

298 hidden layers: the convolutional layers, the pooling layers, and a fully connected layer. The 299 convolutional layers abstract the local information from the data matrix using a kernel. The 300 primary advantage of this layer is the implementation of weight sharing and spatial correlation 301 among neighbors (Guo et al., 2016). The pooling layers are the subsampling layers that reduce the 302 size of the data matrix. A fully connected layer is similar to the traditional neural network added 303 at the final pooling layer after completing an alternate stack of convolutional and pooling layers.

\subsubsection{Long short-term memory (LSTM)}

An LSTM network is a special form of recurrent neural network that stores sequence data for an extended period (Hochreiter and Schmidhuber, 1997). The LSTM structure has three gates:

307 an input gate, an output gate, and a forget gate. The model regulates all these three gates and 308 determines how much data from previous time steps must be stored and transferred to the next 309 steps. The input gate controls the input data at the current time as follows:

$$
a_{l}^{t}=\sum_{i=1}^{I} w_{i l} x_{i}^{t}+\sum_{h=1}^{H} w_{h l} b_{h}^{t-1}+\sum_{c=1}^{C} w_{c l} s_{c}^{t-1} ; b_{l}^{t}=f\left(a_{l}^{t}\right)
$$


311 Where $x_{i}^{t}=$ the input received from the $i^{\text {th }}$ node at time $t ; b_{h}^{t-1}=$ the result of the $h^{\text {th }}$ node at time

$312 t-1 ; s_{c}^{t-1}=$ the cell state (i.e., memory) of the $c^{\text {th }}$ node at time $t$ - 1 . The symbol ' $w$ ' represents the

313 weight between nodes, and the $f$ is the activation function. The output gate transfers the current

314 value from Eq. (5) to the output node, Eq. (6). Then, at the final stage, the current value is stored

315 as the cell state in the forget gate, Eq. (7).

$$
\begin{aligned}
& a_{w}^{t}=\sum_{i=1}^{I} w_{i w} x_{i}^{t}+\sum_{h=1}^{H} w_{h w} b_{h}^{t-1}+\sum_{c=1}^{C} w_{c w} s_{c}^{t-1} ; b_{w}^{t}=f\left(a_{w}^{t}\right) \\
& a_{\emptyset}^{t}=\sum_{i=1}^{I} w_{i \emptyset} x_{i}^{t}+\sum_{h=1}^{H} w_{h \emptyset} b_{h}^{t-1}+\sum_{c=1}^{C} w_{c \emptyset} s_{c}^{t-1} ; b_{\emptyset}^{t}=f\left(a_{\emptyset}^{t}\right)
\end{aligned}
$$

\subsubsection{Gated recurrent unit (GRU) network}

319 The GRU network is an LSTM variant having only two gates, such as reset and update gates (Dey 320 and Salem, 2017). The implementation of this network can be represented by the following 321 equations, Eq. (14-17):

$$
\begin{aligned}
& z=\sigma\left(W_{z} x_{t}+U_{z} h_{t-1}+b_{z}\right) \\
& r=\sigma\left(W_{r} x_{t}+U_{r} h_{t-1}+b_{r}\right) \\
& m=\varnothing\left(W_{m} x_{t}+U_{m}\left(h_{t-1} \cdot r\right)+b_{m}\right) \\
& h_{t}=(1-z) h_{t-1}+z \cdot m
\end{aligned}
$$

326 where $\sigma=$ the sigmoidal activation function; $x_{t}=$ the input value at time $t ; h_{t-1}=$ the output value

327 at time $t-1$; and the $W_{z}, U_{z}, W_{r}, U_{r}, W_{m}, U_{m}$ are the weight matrices for each gate and cell state.

328 The symbols $r$ and $z$ represent the reset and update gates, respectively. $\emptyset$ is the activation function, 329 and the dot [.] represents the element-wise dot product. 


\subsubsection{CLSTM (or CNN-LSTM) Hybrid Model}

In this paper, a deep learning method using optimization techniques is constructed on top

333 of a forecast model framework. This study demonstrates how the CNN-LSTM (CLSTM) model,

334 comprised of four-layered CNN, can be effectively used for UVI forecasting. The CNN is

335 employed to integrate extracted features to forecast the target variable (i.e., UVI) with minimal

336 training and testing error. Likewise, the CNN-GRU (CGRU) hybrid model is prepared for the same

337 purpose.

$338 \quad 2.5$ Optimization techniques

\subsubsection{Ant colony optimization}

Ant colony optimization (ACO) algorithm model is the graphical representation of the real

341 ants' behavior. In general, ants live in colonies, and they forage for food as a whole by

342 communicating with each other using a chemical substance, the pheromones (Mucherino et al.,

343 2015). An isolated ant cannot move randomly; they always optimize their way towards the food

344 deposit to their nests by interacting with previously laid pheromones marks on the way. The entire

345 colony optimizes their routes with this communication process and establishes the shortest path to

346 their nests from feeding sources (Silva et al., 2009). In ACO, the artificial ants find a solution by

347 moving on the problem graph. They deposit synthetic hormone pheromones on the graph so that

348 upcoming artificial ants can follow the pattern to build a better solution. The artificial ants calculate

349 the model's intrinsic mode functions (IMFs) anticipation by testing artificial pheromone values

350 against the target data. The probability of finding the best IMFs increases for every ant because of

351 changing pheromones value throughout the IMFs. The whole process is just like ant's behavior of

352 finding the optimal option to reach the target. The probability $p_{f i}(d)$ of selecting the shortest 
353 distance between the target and the IMFs of the input variable can be mathematically expressed as

354 follows (Prasad et al., 2019):

$355 \quad p_{f i}(d)=\frac{\left(d_{i}+\Delta_{f i}(d)\right)^{2}}{\left(d_{i}+\Delta_{f i}(d)\right)^{2}+\left(d_{i}+\Delta_{f t}(d)\right)^{2}}$

356 Where $f \in\{1,2\}$ denotes decision point, $i$ and $t$ express as short and long distance to the target at

357 an instant $d$ is the total amount of pheromone $\Delta_{f t}(d)$. The probability of the longest path can be

358 determined where $p_{f i}(d)+p_{f t}(d)=1$. The testing update on the two branches is described as

359 follows:

360

$\Delta_{f i}(d)=\Delta_{f i}(d-1)+p_{f i}(d-1) a_{f}(d-1)+p_{k i}(d-1) a_{k}(d-1)$

361

$\Delta_{f t}(d)=\Delta_{f t}(d-1)+p_{f t}(d-1) a_{f}(d-1)+p_{k t}(d-r) a_{k}(d-r)$

362 Where $f, k \in\{1,2\}$ and the value of $r$ represent the remainder in the model. $a_{f}(d)$ denotes the

363 number of ants in the node $f$ at a certain period $d$ is given by:

$364 a_{f}(d)=p_{k i}(d-1) a_{k}(d-1)+p_{k t}(d-r) a_{k}(d-r)$

365 The ACO algorithm is the most used simulation optimization algorithm where myriad artificial 366 ants work in a simulated mathematical space to search for optimal solutions for a given problem.

367 The ant colony algorithm is dominant in multi-objective optimization as it follows the natural 368 distribution and self-evolved simple process. However, with the increase of network information, 369 the ACO algorithm faces various constraints such as local optimization and feature redundancy 370 for selecting optimal pathways (Peng et al., 2018).

\section{$371 \quad$ 2.5.2 Differential evaluation optimization}


372 The differential evolution (DEV) algorithm is renowned for its simplicity and powerful stochastic

373 direct search method. Besides, DEV has proven an efficient and effective method for searching

374 global optimal solutions for the multimodal objective function, utilizing N-D-dimensional

375 parameter vectors (Seme and Štumberger, 2011). It does not require a specific starting point, and

376 it operates effectively on a population candidate solution. The constant value $\mathrm{N}$ denotes the

377 population; in every module, a new generation solution is determined and compared with the

378 previous generation of the population member. It is a repetition process and runs until it reaches

379 the maximum number of generations (i.e., $\mathrm{G}_{\max }$ ). The $G$ defines the generation number of

380 populations which can be written in mathematical proportional order. If the initial population

381 vector is $S_{G}$, then $S_{G}=i_{1, G}, i_{2, G} \ldots \ldots, i_{N P, G}$, and $G=0, \ldots, G_{\max }$

$382 i_{n, G}, n=1,2, \ldots \ldots, N$

383 The initial population $S_{G=0}$ is generated using random within given boundaries, which can be 384 written in the following equation:

$385 i_{j, 0}^{n}=\operatorname{rand}_{j}[0,1]\left(i_{j}^{(U)}-i_{j}^{(L)}\right)+i_{j}^{(L)}, n=1,2, \ldots, N P, j=1,2, \ldots, D$

386 Where $\operatorname{rand}_{j}[0,1]$ is the uniformly distributed number at the interval $[0,1]$, which is chosen a new

387 for each $j$. $D$ represents the boundary condition. In contrast, $(U)$ and $(L)$ represents the upper and

388 lower limit of the boundary vector parameters. For every generation, a new random vector is

389 randomly created, selecting vectors from the previous generation from the following manner:

$390 \quad c_{j, G}^{n}=\left\{\begin{array}{l}i_{j, G-1}^{r}+F\left(i_{j, G-1}^{r}-i_{j, G-1}^{r}\right) \text { ifrand }_{j}[0,1] \leq \\ i_{j, G-1}^{n} \text { otherwise }\end{array}\right\}$ 
391 Where, $r$ is the number of optimizations, $c$ is the candidate vector, $C R \in[0,1]$ and $F \in[0,2]$

392 control parameter. $k$ is the randomly selected index that ensures the difference between the

393 candidate vector and the generation vector. The population for new the new generation $S_{G}$ will be

394 assembled from the vector of the previous generation $S_{G-1}$ and the candidate vectors $c_{j, G}^{n}$ the

395 following equation can describe selection:

$396 G=0, \ldots, G_{\max } ; n=1,2, \ldots \ldots \ldots, N P$

$397 \quad I_{G}^{n}=\left\{\begin{array}{l}c_{G}^{n} \text { if } f\left(c_{G}^{n}\right) \leq f\left(I_{G-1}^{n}\right) \\ I_{G-1}^{n} \text { otherwise }\end{array}\right\}$

398 The process repeats with the following generation population number until it satisfies the pre-

399 defined objective function.

\subsubsection{Particle swarm optimization}

401 The particle swarm optimization (PSO) method was developed for continuous non-linear

402 functions optimization having roots in artificial life and evolutionary computation (Kennedy and

403 Eberhart, 1995). The method was constructed using a simple concept that tracks each particle's

404 current position in the swarm by implementing a velocity vector for particle's previous to the new

405 position. However, the movement of the particles in the swarm depends on the individuals'

406 external behavior. Therefore, the process is very speculative, uses each particle's memory for

407 calculating new position, and gained knowledge by the swarm as a whole. Nearest neighbor

408 velocity matching and craziness, eliminating ancillary variables and incorporate multidimensional

409 search and acceleration by distance, were the precursor of PSO algorithm simulation (Eberhart and

410 Shi, 2001). Each particle in the simulation coordinates in the n-dimensional space calculation and

411 responds to the two quality factors called 'gbest' and 'pbest'. gbest represents the best location

412 and value of particle in the population globally, and pbest represents the best-fitted solution 
413 achieved by the particle so far in the population swarm. Thus, at each time step in the swarm, the

414 PSO concept stands, each particle changing its acceleration towards its two best quality factor

415 locations. The acceleration process begins by separating random numbers and presenting the

416 optimal 'gbest' and 'pbest' locations. The basic steps for the PSO algorithm are given below,

417 according to (Eberhart and Shi, 2001):

418 1. The process starts with initializing sample random particles with random velocities and 419 locations on $n$-dimensions in the design space.

4202 . The velocity vector for each particle in the swarm is carried out in the next step as the initial $421 \quad$ velocity vector value.

422 3. Plot the velocity vector value and compare particle fitness evaluation with particle's pbest.

423 If the new value is better than the initial value, update the new velocity vector value as 424 pbest and previous location equal to the current location in the design space.

4254 . In this step, compare the fitness evaluation with the particles' overall previous global best.

426 If the current value is better than gbest, update it to a new gbest value and location.

4275 . The velocity and position of the particle can be changed according to the equations:

$$
\begin{aligned}
& v_{n d}=v_{n d}+m_{1} * \operatorname{rand}(x) *\left(p_{n d}-z_{n d}\right)+m_{2} * \operatorname{Rand}(x) *\left(p_{g d}-z_{n d}\right) \\
& z_{n d}=z_{n d}+v_{n d}
\end{aligned}
$$

6. Repeat step 2 and continue until the sufficiently fitted value and position are achieved.

431 Particle swarm optimization is well known for its simple operative steps and performance for 432 optimizing a wide range of functions. PSO algorithm can successfully solve the design problem 433 with many local minima and deal with regular and irregular design space problems locally and 434 globally. Although PSO can solve problems more accurately than other traditional gradient-based 435 optimizers, the computational cost is higher in PSO (Ventor and Sobieszczanski-Sobieski, 2003). 


\subsubsection{Genetic algorithm}

437 The genetic algorithm (GA) is a heuristic search method based on natural selection and 438 evolution principles and concepts. This method was introduced by John Holland in the mid439 seventies, inspired by Darwin's theory of descent with modification by natural selection. To 440 determine the optimal set of parameters GA mimics the reproduction behavior of the biological 441 populations in nature. It has been proven effective for the selection process in solving cutting-edge

442 optimization problems. It can also handle regular and irregular variables, non-traditional data 443 partitioning, and non-linear objective functions without requiring gradient information (Hassan et 444 al., 2004). The basic steps for the PSO algorithm are given below:

1. The determination of the maximum outcomes from an objective function, the genetic algorithm uses the following function (Mayer and Lobet, 2018):

where $n$ is the number of decision variables $y_{i} \in\left[y_{i}^{\min }, y_{i}^{\max }\right]$ with a discretization step $\delta y_{i}$. The

449 initial boundary conditions $y_{i}^{\min }, y_{i}^{\max }$ determined in the beginning of the simulation. $\delta y_{i}$ is the 450 determines the physical parameters $y_{i}$ performances in the experiment. These decision variables 451 are represented by a sequence of binary digits (GENES).

2. The decisions variables are given within initial boundary conditions $y_{i}=y_{i}^{\text {min }}+$ $(G E N E i) * \delta y_{i}$, where GENE $i \in\left[0,2^{n_{i}}-1\right]$ refers to the value of GENES. $n_{i}$ is the bit length of each GENE, which is the first integer where $y_{i}^{\min }+2^{n_{i}}-1 * \delta y_{i} \geq y_{i}^{\max }$. The total number of bits in each DNA refers $n_{\text {sum }}=\sum_{i=1}^{n} n_{i}$. The algorithm process starts with a random selection of objectives. After evaluation of each objective in the fitness function $f=f\left(\left(y_{1}+y_{2}\right), \ldots\left(y_{n}+y_{n+1}\right)_{n}\right)$, and rank them from best to worst. 
458 The genetic similarity determines the selection progress indicator. These random individual

459 objectives with rank are transferred to the next generation. The remaining individuals participate

460 in the steps of selection, crossover, and mutation. The individual objective parent selection process

461 can happen several times, and this can be achieved by many different schemes, such as the roulette-

462 wheel ranked method. For any pair of objective parents' selection, crossover, and mutation process

463 of next-generation is defined. After that, the fitness $f$ of all individuals scheduled for the next

464 generation is evaluated. This process repeats from generation to generation until a termination

465 criterion is met.

GA methodology is quite similar to another stochastic searching algorithm PSO. Both

467 methods begin their search from a randomly generated population of designs that evolve over 468 successive generations. They do not require any specific starting point for the simulation. The first 469 operator is the "selection" procedure similar to "Survival for the Fittest" principle. The second 470 operator is the "Crossover" operator, which mimics mating in a biological population. Both 471 methods use the same convergence criteria for selecting the optimal solution in the problem space

472 (Hassan et al., 2004). However, GA differs in two ways from the most traditional optimization 473 methods. First, GA does not operate directly on the design parameter vector but a symbolic 474 parameter known as a chromosome. Second, it optimizes the whole design chromosomes at once, 475 unlike other optimization methods single chromosome at a time (Weile and Michielssen, 1997).

\subsubsection{Complete Ensemble Empirical Mode Decomposition with Adaptive Noise (CEEMDAN)}

477 The complete ensemble empirical mode decomposition with adaptive noise (CEEMDAN) 478 decomposition approach initiates by discretizing the $n$-length predictors of any model $\chi(t)$ into 479 IMFs (intrinsic model functions) and residues to conform with tolerability. However, to ensure no 480 information leakage in the IMFs and residues, the decomposition is performed separately by taking 
481 training and testing subsets. The actual IMF is produced by taking the mean of the Empirical mode 482 decomposition (EMD)-grounded IMFs across a trial and combining white noise to model the 483 predictor-target variables. The CEEMDAN is used in machinery, electricity, and medicine such as 484 impact signal denoising, daily peak load forecasting, health degradation monitoring for rolling 485 bearings, friction signal denoising combined with mutual information (Li et al., 2019).

486 The CEEMDAN process is as follows:

487 Step 1: The decomposition of $p$-realizations of $\chi[n]=\varepsilon_{1} \omega^{p}[n]$ using EMD to develop their first 488 intrinsic approach, as explained according to the equation:

$$
\widehat{I M F_{1}}[n]=\frac{1}{p} \sum_{p=1}^{P} I M F_{1}^{p}[n]=I \bar{M} F_{1}[n]
$$

490 Step 2: Putting $k=1$, the $1^{\text {st }}$ residue is computed following Eq. (1).

$$
\operatorname{Res}_{1}[n]=\chi[n]-\widehat{I M F_{1}}[n]
$$

492 Step 3: Putting $k=2$, the $2^{\text {nd }}$ residual is obtained as

$$
\widehat{I M F_{2}}[n]=\frac{1}{p} \sum_{p=1}^{P} E_{1}\left(r_{1}[n]+\varepsilon_{1} E_{1}\left(\omega^{p}[n]\right)\right)
$$

494 Step 4: Setting $k=2 \ldots K$ calculates the $k^{\text {th }}$ residue as.

$$
\operatorname{Res}_{k}[n]=\operatorname{Res}_{k-1}[n]-\widehat{I M F_{k}}[n]
$$

496 Step 5: Now, we decompose the realizations $\operatorname{Res}_{k}[n]+\varepsilon_{1} E_{1}\left(\omega^{p}[n]\right)$, Here, $k=1, \ldots K$ until 497 their first model of EMD is reached; Here the $(k+1)$ is

$$
\widehat{I M F}_{(k+1)}[n]=\frac{1}{p} \sum_{p=1}^{P} E_{1}\left(r_{k}[n]+\varepsilon_{k} E_{k}\left(\omega^{p}[n]\right)\right)
$$


499 Step 6: Now, the $k$ value is incremented, and steps 4-6 are repeated. Consequently, the final 500 residue is achieved:

$501 \quad \operatorname{RES}_{k}[n]=\chi[n]-\sum_{k=1}^{K} \widehat{I M F_{k}}$

502 Here, $K$ is defined as the limiting case (i.e., the highest number of modes). To comply with the

503 replicability of the earliest input, $\chi[n]$., the following is performed for the CEEMDAN approach.

$504 \chi[n]=\sum_{k=1}^{K} \widehat{I M F_{k}}+\operatorname{RES}_{k}[n]$

\subsection{Model implementation procedure}

507 It is crucial to optimize the objective model's architecture to incorporate the relationship between

508 predictors and model. A multi-phase CNN-GRU and GRU model is included, built using Python-

509 based deep learning packages such as TensorFlow and Keras. A total of nine statistical metrics

510 was used to investigate the forecasting robustness of the models that have been integrated. The

511 model was powered by an Intel i7 @ 3.6GHz processor and 16 GB of memory. Deep learning

512 libraries like Keras (Brownlee, 2016; Ketkar, 2017) and TensorFlow (Abadi et al., 2016) were

513 used to demonstrating algorithms for the proposed models. In addition, packages like matplotlib

514 (Barrett et al., 2005) and seaborn (Waskom, 2021) were used for visualization.

515 The determination of the model's valid predictors does not have any precise formula.

516 However, the literature suggests three methods, i.e., trial-and-error, the autocorrelation function

517 (ACF) and partial autocorrelation function (PACF), and the cross-correlation function (CCF)

518 approaches, for selecting lagged UVI memories and predictors to make an optimal model. In this

519 study, the PACF was used to determine significant antecedent behavior in terms of the lag of UVI

520 (Tiwari and Adamowski, 2013; Tiwari and Chatterjee, 2010). Figures 6b and 7b demonstrated the 
521 PACF for UVI time series showing the antecedent behavior in terms of the lag of UVI and 522 decomposed UVI (i.e., $\mathrm{IMF}_{\mathrm{n}}$ ) where antecedent daily delays are apparent. Generally, the CCF 523 selects the input signal pattern based on the predictors' antecedent lag (Adamowski et al., 2012). 524 The CCF determined the predictors' statistical similarity to the target variable (Figures 6a and 7a). 525 A set of significant input combinations was selected after evaluating each predictor's $r_{\text {cross }}$ with 526 UVI. The figure shows that the highest correlation between $\mathrm{p}$ data and UVI was found for all 527 stations at lag zero (i.e., $r_{\text {cross }}=0.22-0.75$ ). AOD and GBI both demonstrated significant $r_{\text {cross }}$ 528 from 0.65 to 0.80 and 0.68 to 0.75 , respectively. Some predictors with insignificant lags such as 529 AO, CT, and OTC were also considered to increase the predictors' diversity. The CCF with UVI 530 with predictors significantly varied for all other stations. However, selecting lags from the cross531 correlation function is identical to that used in the objective stations.

As mentioned, the CEEMDAN method was used to decompose the data sets. The daily 533 time series of UVI data and predictor variables were decomposed into respective daily IMFs and 534 a residual component using CEEMDAN procedures. The example of the IMFs and the residual 535 component of the respective CEEMDAN is shown in Figure 3. PACF was applied to the daily 536 IMFs and residual component time series generated in the above process. An individual input 537 matrix was created for each IMF, and the residual component was made up based on the significant 538 lagged memory with that of IMF of target UVI. These separate input matrices were used to forecast 539 the respective future IMFs and the residual component. Next, the anticipated IMFs and residuals 540 were combined to produce daily forecasts of UVI values. Note that the CEEMDAN 541 transformations are completely self-adaptive and data-dependent multi-resolution techniques. As 542 such, the number of IMFs and the residual component generated are contingent on the nature of 543 the data. 
545 minimize the scaling effect of different variables as follows:

$546 \quad U_{\text {norm }}=\frac{U-U_{\min }}{U_{\max }-U_{\min }}$

547 In Eq. (30), $U$ is the respective predictors, $U_{\min }$ is the minimum value for the predictors, $U_{\max }$ is

548 the maximum value of the data and $U_{\text {norm }}$ is the normalized value of the data. After normalizing

549 the predictor variables, the data sets were partitioned: $70 \%$ of the data sets were considered training

550 data, $15 \%$ were used for testing, and the remaining $15 \%$ of the data sets were considered validation

551 data. The LSTM model was followed by developing a hybrid LSTM model with 3-layered CNN

552 and 4-layered LSTM, as illustrated in Figure 2. Using the conventional models, the traditional

553 antecedent lagged matrix of the daily predictors' variables was applied. The prior application of

554 the optimization algorithm was made before using CCF and PACF and before significant

555 predictors being removed from the model. Table 2 shows the selected predictors using four

556 optimization techniques in association with the UVI.

$557 \quad 2.7$ Model performance assessment

558 In this study, the effectiveness of the deep learning hybrid model was assessed using a variety of 559 performance evaluation criteria, e.g., Pearson's Correlation Coefficient $(r)$, root mean square error 560 (RMSE), Nash- Sutcliffe efficiency (NS) (Nash and Sutcliffe, 1970), and mean absolute error 561 (MAE). The relative RMSE (denoted as RRMSE) and relative MAE (denoted as RMAE) were used 562 to explore the geographic differences between the study stations.

564 evaluate the effectiveness of a predictive model. When the error distribution in the tested data is 565 Gaussian, the root mean square error (RMSE) is a more appropriate measure of model performance 
566 than the mean absolute error (MAE) (Chai and Draxler, 2014), but for an improved model

567 evaluation, Legates-McCabe's $(L M)$ Index are used as more sophisticated and compelling

568 measures (Legates and McCabe, 2013; Willmott et al., 2012). Mathematically, the metrics are as

569 follows:

$570 \quad$ i) Correlation coefficient $(r)$ :

$571 \quad r=\left\{\frac{\sum_{i=1}^{N}\left(U V I_{o b s}-\overline{U V I}_{o b s}\right)\left(U V I_{f o r}-\overline{U V I}_{f o r}\right)}{\sqrt{\sum_{i=1}^{N}\left(U V I_{o b s}-\overline{U V I}_{o b s}\right)^{2} \sum_{i=1}^{N}\left(U V I_{f o r}-\overline{U V I}_{f o r}\right)^{2}}}\right\}^{2}$

572 ii) Mean absolute error $(M A E)$ :

$573 \quad \mathrm{MAE}=\frac{1}{\mathrm{~N}} \sum_{\mathrm{i}=1}^{\mathrm{N}}\left|U V I_{\text {for }-} U V I_{\mathrm{obs}}\right|$

574 iii) Root mean squared error (RMSE):

$575 \quad$ RMSE $=\sqrt{\frac{1}{N} \sum_{\mathrm{i}=1}^{\mathrm{N}}\left(\mathrm{UVI}_{\mathrm{for}-} U V I_{\mathrm{obs}}\right)^{2}}$

576 iv) Nash-Sutcliffe Efficiency $(N S)$ :

$\left.577 \quad \mathrm{NSE}=1-\left[1-\frac{\sum_{\mathrm{i}=1}^{\mathrm{N}}\left(U V I_{\text {for }}\right)^{2}}{\sum_{\mathrm{i}=1}^{\mathrm{N}}\left(U V I_{o b s}-\overline{U V I}_{f o r}\right)^{2}}\right]\right)$

578 v) Legates-McCabe's Index $(L M)$ :

$579 L M=1-\left[\frac{\sum_{\mathrm{i}=1}^{\mathrm{N}} \mid U V I_{\mathrm{for}-U V I_{\mathrm{obs}} \mid}}{\sum_{\mathrm{i}=1}^{\mathrm{N}}|| U V I_{\mathrm{obs}-} \overline{U V I}_{\mathrm{obs}} \mid}\right]$

580 vi) Relative Root Mean Squared Error (RRMSE, \%):

$581 \quad R R M S E(\%)=\frac{\sqrt{\frac{1}{N} \sum_{\mathrm{i}=1}^{\mathrm{N}}\left(U V I_{\mathrm{for}-} U V I_{\mathrm{obs}}\right)^{2}}}{\frac{1}{N} \sum_{i=1}^{N}\left(U V I_{\text {obs }}\right)} \times 100$

582 vii) Relative Mean Absolute Error (RMAE, \%): 
$583 \quad R M A E(\%)=\frac{\frac{1}{\mathrm{~N}} \sum_{\mathrm{i}=1}^{\mathrm{N}}\left|U V I_{\mathrm{for}}-U V I_{\mathrm{obs}}\right|}{\frac{1}{N} \sum_{i=1}^{N}\left(U V I_{\text {obs }}\right)} \times 100$

In Eq. (31-37), $U V I_{\text {obs }}$ and $U V I_{\text {for }}$ represents the observed and forecasted values for $i^{\text {th }}$ test

585 value; $\overline{U V I}_{o b s}$ and $\overline{U V I}_{f o r}$ refer to their averages, accordingly, and $N$ is defined as the number of 586 observations, while the $C V$ stands for the coefficient of variation.

\section{3. Results}

588

This section describes results obtained from the proposed hybrid deep learning model (i.e.,

590 CEEMDAN-CLSTM) and other hybrid models (i.e., CEEMDAN-CGRU, CEEMDAN-LSTM,

591 CEEMDAN-GRU, CEEMDAN-DT, CGRU, and CLSTM), and the standalone LSTM, GRU, DT,

592 MLP, and SVR models. The evolutionary algorithms (i.e., ACO, DEV, GA, and PSO) were

593 incorporated to obtain the optimum features in model building. Seven statistical metrics from Eqs.

594 (31) to (37) were used to analyze the models in the testing dataset and visual plots to justify the

595 forecasted results' justification.

\subsection{The evaluation of hybrid and standalone models}

The hybrid deep learning model (i.e., CEEMDAN-CLSTM) demonstrated high $\mathrm{r}$ and NS

598 values and low RMSE and MAE compared to their standalone models (Table 3). The best overall

599 performance was recorded using the CEEMDAN-CLSTM model with the Genetic Algorithm with

600 the highest correlation (i.e., $r=0.996$ ), the highest data variance explained (i.e., NS = 0.997), and

601 the lowest errors (i.e., RMSE $=0.162$ and MAE $=0.119$ ). The performance was followed by the 602 same model with Partial Swarm Optimisation (i.e., $r \approx 0.996 ; \mathrm{NS} \approx 0.992$; RMSE $\approx 0.216$; MAE $603 \approx 0.163$ ) and Ant Colony Optimisation (i.e., $\mathrm{r} \approx 0.996 ; \mathrm{NS} \approx 0.993 ; \mathrm{RMSE} \approx 0.220$; MAE $\approx 0.165$ ).

604 The single deep learning models (i.e., LSTM and GRU) performed better than the single machine 
605 learning models (i.e., DT, SVR, and MLP). Moreover, the hybrid deep learning models without a

606 CNN (i.e., CEEMDAN-GRU and CEEMDAN-GRU) also demonstrated higher forecasting 607 accuracy (i.e., $r=0.973-0.993 ; \mathrm{RMSE}=0.387-0.796$ ) in comparison with standalone deep 608 learning models ( i.e., $\mathrm{r} \approx 0.959-0.981 ; \mathrm{RMSE} \approx 0.690-0.986$ ). The following models' 609 performance is then predicted by the CNN-GRU, CEEMDAN-GRU, and GRU models in that 610 order.

\section{$611 \quad 3.2$ The selection of the best model}

RRMSE and LM for all tested models were used to assess the robustness of the proposed

613 hybrid models as well as for comparisons. The magnitude of RRMSE (\%) and LM for the objective

614 model (CEEMDAN-CLSTM) shown in Figure 6 indicates that the proposed hybrid model

615 performed significantly better than other benchmark models. The RRMSE and LM values ranged

616 between 2 and 3.5 percent and between 0.982 and 0.991 , respectively. The performance indices

617 (i.e., RRMSE and LM) using four optimization algorithms were higher for the CEEMDAN-CGRU

618 model. Overall, the CEEMDAN-CLSTM model with the GA optimization methods provided the

619 best performance (i.e., $\mathrm{RRMSE}=\sim 2.0 \%$; $\mathrm{LM}=0.991$ ), indicating its high efficiency in forecasting

620 the future UV-Index a higher degree of accuracy.

621 A precise comparison of forecasted and observed UVI can also be seen by examining the 622 scatterplot of forecasted ( $\left.\mathrm{UVI}_{\text {for }}\right)$ and observed $\left(\mathrm{UVI}_{\mathrm{obs}}\right) \mathrm{UVI}$ for four optimization algorithms (i.e.,

623 ACO, PSO, DEV, and GA) (Figure 7). Here, scatter plots showed the coefficient of determination $624\left(\mathrm{r}^{2}\right)$ and a least-square fitting line, along with the equation for UVI and an observed UVI close to 625 the forecasted UVI. As demonstrated in Figure 7, it also appears that the proposed hybrid model 626 performed better when compared with other applied models. However, among the four 
627 optimization techniques applied, the hybrid deep learning model (i.e., CEEMDAN-CLSTM)

628 optimized with the GA outperformed the other models in forecasting the UVI. The hybrid

629 CEEMDAN-CLSTM model calculated magnitudes from the GA, which came the closest to unity,

630 with an $\mathrm{m} \mid \mathrm{r}^{2}$ of $0.976 \mid 0.995$ in pairs. The performance is followed by ACO and DEV algorithms

631 with a potential pair (ACO: 0.975|0.995; DEV: 0.966|0.994). The outliers (i.e., the extremes) are

632 closer to the fitted line, while the y-intercept (i.e., the starting point) is approximately 0.05 units

633 away from zero (0) using the GA method. The other models had outliers, resulting in their

634 intercepts deviating from the ideal value. In conclusion, the CEEMDAN-CLSTM model

635 performed the best for the GA.

636 The proposed hybrid deep learning model (i.e., CEEMDAN-CLSTM) was further assessed 637 employing the ECDFs of absolute forecast error ( $|\mathrm{FE}|)$ (Figure 8). Total 95\% forecasted values 638 using the CEEMDAN-CLSTM model with GA demonstrated a small error ranged between 0.01 639 and 0.299 , with a substantially larger error for the CCGRU model (i.e., 0.477), followed by the 640 CLSTM model (i.e., 0.626) and CGRU (i.e., 1.104). For the other optimization algorithms, nearly 641 the same level of performance was observed. Predictions ranging between the $95^{\text {th }}$ and $98^{\text {th }}$ 642 percentile were preferred over objective models, which performed best in the current forecast. A 643 hybrid CLSTM model was found to be the most accurate for upcoming extra-terrestrial intelligent 644 beings. However, Figure 9 showed the effect of applying CEEMDAN as a feature extraction 645 method of data on the percent change in RMAE values within the testing phase of UVI forecast 646 incrementally. The contribution of the data decomposition method (i.e., CEEMDAN) was 647 significant in the model implementation. The decrement of RMAE in percent using GA was found 648 between $17 \%$ to $63 \%$, whereas the CLSTM showed the highest percentage of decrement (i.e., $64963 \%)$. Moreover, the PSO optimized model showed that the RMAE (\%) values with the deep 
650 learning model appeared to decrease by $\sim 2$ to $60 \%$, and the lowest decreasing RMAE was found 651 for the ACO algorithm with a reduction $\sim 3 \%$ to $36 \%$. However, the CLSTM model using four 652 optimization methods showed the highest improvement among all the deep learning approaches 653 that reduced the RMSE from $36 \%$ to $63 \%$. It is worth mentioning that the percent increase in $654 R M A E$ was $\sim 83 \%$ for the DEV algorithm using the SVR method. Overall, the CEEMDAN, as a 655 data decomposition algorithm for UVI forecasting with four optimization algorithms, showed 656 significant improvement over the testing phase.

After additional analysis, the forecasted-to-observed UVI and absolute forecasting errors 658 are displayed in Figure 10. The absolute forecasted error has a maximum dispersion of $(|\mathrm{FE}|=$ $\left.659\left|\mathrm{UVI}_{\mathrm{for}}-\mathrm{UVI}_{\mathrm{obs}}\right|\right)$. The box plot demonstrated the data dispersal of the observed and forecasted 660 UVI from the proposed deep learning approaches and other comparing models. Figure 10 provides 661 a clear visualization of the data concerning quartiles distinctly outliers. The lower end of the plot 662 lies between the lower quartile ( $25^{\text {th }}$ percentile) and upper quartile $\left(75^{\text {th }}\right.$ percentile). It is evident 663 that the median of the forecasted and the observed UVI for the CEEMDAN-CLSTM model with 664 the GA optimization. Moreover, the DEV-based CEEMDAN-CLSTM model showed identical forecasting to the 666 GA-based CEEMDAN-CLSTM model with a slight variation. A more in-depth inspection of the 667 absolute forecasted error (|FE|) from the hybrid CEEMDAN-CLSTM model for two optimizations 668 (i.e., GA and DEV) further strengthens the suitability of the hybrid CLSTM approach in 669 forecasting the UVI of Perth station of Australia with the narrowest distribution in comparison 670 with other models. A significant percentage (98\%) of the $|\mathrm{FE}|$ in the first error brackets $671(0<|\mathrm{FE}|<0.15)$ was observed for the GA-based CEEMDAN-CLSTM model, while for the DEV672 based model, the percentage is $95 \%$. 
With the help of a time series plot, we can better understand forecasting ability and refine

674 the proposed model, taking it from standalone to hybrid model. The time series plot of forecasted

675 and observed UVI using CEEMDAN-CLSTM optimized by four optimization methods is depicted

676 in Figure 11. The results showed that the proposed GA-based CEEMDAN-CLSTM model is close

677 to the observed UVI, indicating that the model has high predictive accuracy. The application of

678 the GA in the model optimization resulted in a significant improvement in forecasted UVI. For

679 other algorithms that use the CEEMDAN-CLSTM model, it is discovered that the forecasted UVI

680 is accurate when compared to the other optimization methods.

681 Finally, Figure 12 presents a comprehensive interpretation by illustrating the absolute

682 forecasting error frequency distributions ( $|\mathrm{FE}|)$ using all GA-based models for Perth stations of

683 Australia. It is apparent from Figure 12 that the CEEMDAN-CLSTM model provided significantly

684 improved distributions with the maximum $98 \%$ forecasting error $(|\mathrm{FE}|)$ within the first error

685 brackets $(0<|\mathrm{FE}|<0.10)$. It is also noteworthy that the CEEMDAN-CGRU model showed a higher

686 percentage of $|\mathrm{FE}|$ between 0 and 0.25 of all forecasting yielded a considerably small error and the

687 remaining $15 \%$ of simultaneously produced forecasting error between 0.25 and 1.0 . The highest

688 forecasted error was found for machine learning models when the $|\mathrm{FE}|$ value for all models (i.e.,

689 SVR, MLP, and DT) was considered.

690

691 4. Discussion

692

The establishment of robust predictive modelling of the UV index and physical

693 interpretation is critical for various practical applications, such as helping policymakers in their 
694 daily health impact assessment. These systems emulate how a human expert would solve a 695 complex forecasting problem by reasoning through a set of UVI-related predictors rather than

696 through conventional or procedural methods. These methods warrant continuous measurement of 697 irradiance or radiative transfer models, which are tedious (as discussed in the introduction) and 698 often inaccurate. This study demonstrated the efficacy of hybrid deep learning methods in 699 forecasting UVI on a near real-time horizon. The study site was in Perth, Western Australia, 700 Australia, where skin cancer is significantly high. An accurate forecasting system in this region is 701 therefore essential.

To function effectively, alert systems must generate accurate irradiance forecasts, but UVI 703 is generally determined by many factors (i.e., the solar zenith, altitude, cloud fraction, aerosol and 704 optical properties, albedo, and vertical ozone profile) (Deo et al., 2017). The study extensively 705 utilized four optimization techniques (i.e., GA, ACO, DEV, and PSO) to have optimum predictors 706 used in UVI forecasting. The incorporated predictors from three distinct data sets (i.e., SILO, 707 MODIS, and CI) were optimized. The optimization techniques selected a diversified list of 708 variables except for RMM1 and RMM2, as four algorithms select them both. The predictors like 709 ozone total column, AOD, and cloud fraction were significant using the GA algorithm. In most 710 cases, the hydro-meteorological variables were insignificant by all four algorithms that agree with 711 UV concentration's general concept. The objective algorithm (i.e., GA) selected SOI, GBI, AAO, 712 Nino4, Nino12, RMM1, and RMM2 as potential predictors as well. The ground-based 713 measurements and modelling studies are essential (Alados et al., 2007, 2004), but are challenging 714 to implement in practice. Furthermore, secondary factors affecting UV levels (i.e., clouds or 715 aerosols) are rarely known with sufficient precision. Considering the practical feasibility, an 716 algorithm that is data-efficient, simple to develop, flexible, and user-friendly should be considered 
717 as a viable alternative for information (Igoe et al., 2013a, 2013b; Parisi et al., 2016). Therefore,

718 our developed forecasting model will play a vital role in the decision-makers to adopt prompt

719 measures without difficulties.

720 The proposed hybrid deep learning network (i.e., CEEMDAN-CLSTM) for predicting

721 surface UV radiation also demonstrated low errors in forecasting, i.e., showing around $10 \%$ error

722 for the next-day forecast and 13-16\% error for 7-day up to the 4-week forecast. This further affirms

723 that the quantitative UV forecast is appropriate for heliotherapy applications, which tolerates up

724 to $10-25 \%$ error levels. The CEEMDAN-CLSTM's performance is competitive on UV data from

725 multiple regions. Thus, the CEEMDAN-CLTSM model can be adapted to forecast other useful

726 UV action spectra, such as vitamin D production and erythemal UV index. A key limitation of

727 machine learning is its overfitting tendency on the training dataset and often does not generalize

728 well to other datasets from different distributions. In the context of UV forecasting, this dictates

729 that the model must be retrained with data from the weather station to be used for that geographic

730 region. In a geographical region with the highly variable weather condition, such as London in

731 2019, artificial neural network models’ performance dropped significantly (Raksasat et al., 2021).

732 This capability of the model to extract seasonal patterns may also explain why the addition of

733 ozone, cloud fraction, and AOD information significantly improved the performance of

734 CEEMDAN-CLSTM, particularly when the GA algorithm was applied.

\section{5. Conclusion}

736 This study conducted a daily UV Index forecasting at Perth station using aggregated 737 significant antecedent satellite-driven variables associated with UV irradiance. The forecasting 738 was made using a novel hybrid deep learning model (i.e., CEEMDAN-CLSTM) and compared 
739 with other benchmark models such as LSTM, GRU, DT, SVR, etc. Four optimization methods 740 were employed to extract the crucial features of the response variable (i.e., the UVI). After

741 applying the proposed model and benchmarked models, the model's merits were evaluated using

742 different statistical metrics (e.g., r, WI, MAE, NS, and LM), graphical plots, and relevant

743 discussions. The key findings are summarized as follows:

- The CEEMDAN-CLSTM hybrid model demonstrated excellent forecasting ability compared to its counterpart models.

- The GA optimization algorithm is appeared to be an attractive option for selecting mechanistically meaningful features of the dependable variable compared to the other three optimization techniques.

- The performance metrics showed that the GA and CEEMDAN-optimized models had better performance and higher efficiency metrics (i.e., r, NS, and LM) and lower error metrics (i.e., MSE and RMSE).

- However, in UVI forecasting, the standalone models' (i.e., LSTM, GRU, DT, and SVR) performances were poor compared to the proposed hybrid model.

Adapted to an Australian climate in the sub-tropics during peak summer-time conditions, applying a CLSTM model to forecast the UVI is a novel deep learning approach. The forecasts derived from our data were within one UVI unit of the actual measured values indicating the remarkable forecasting capability. Therefore, this data-driven model would be of tremendous help for the decision-makers to promptly protect public health without delay. It has the tremendous potential to be adopted by a more significant segment of the community, particularly children and 
the elderly facing a greater risk of developing skin cancer (i.e., melanoma) in the Australian region

761 and worldwide.

762

763

764

765

766

767

768

769

770

771

772

\section{3}

774

775

776

777

778

779

780

781

782

783

784

785

786

787

788

789

790

791

792

\section{Acknowledgement}

We would like to thank the Australian Radiation Protection and Nuclear Safety Agency for providing the data. This study did not receive any external funding.

\section{Credit authorship contribution statement}

A. A. Masrur Ahmed: Writing - original draft, Conceptualization, Methodology, Software, Model development, and application. Mohammad Hafez Ahmed: Conceptualization, Model Application, Writing - draft, review \& editing. Sanjoy Kanti Saha: Writing - review \& editing. Oli Ahmed: Data Collection, Writing - review \& editing, Ambica Sutradhar: Data Collection, Writing - review \& editing

\section{Reference}

Abadi, M., Barham, P., Chen, J., Chen, Z., Davis, A., Dean, J., Devin, M., Ghemawat, S., Irving, G., Isard, M., Kudlur, M., Levenberg, J., Monga, R., Moore, S., Murray, D.G., Steiner, B., Tucker, P., Vasudevan, V., Warden, P., Wicke, M., Yu, Y., Zheng, X., 2016. TensorFlow: A System for Large-Scale Machine Learning. Presented at the 12th \{USENIX\} Symposium on Operating Systems Design and Implementation (\{OSDI\} 16), pp. 265-283.

Adamowski, J., Chan, H.F., Prasher, S.O., Ozga-Zielinski, B., Sliusarieva, A., 2012. Comparison of multiple linear and non-linear regression, autoregressive integrated moving average, artificial neural network, and wavelet artificial neural network methods for urban water demand forecasting in Montreal, Canada. Water Resources Research 48. https://doi.org/10.1029/2010WR009945

Ahmed, A.A.M., Deo, R.C., Ghahramani, A., Raj, N., Feng, Q., Yin, Z., Yang, L., 2021. LSTM integrated with Boruta-random forest optimiser for soil moisture estimation under RCP4.5 and RCP8.5 global warming scenarios. Stoch Environ Res Risk Assess. https://doi.org/10.1007/s00477-021-01969-3

Ahmed, M.H., Lin, L.-S., 2021. Dissolved oxygen concentration predictions for running waters with different land use land cover using a quantile regression forest machine learning technique. Journal of Hydrology 597, 126213. https://doi.org/10.1016/j.jhydrol.2021.126213 
Alados, I., Gomera, M.A., Foyo-Moreno, I., Alados-Arboledas, L., 2007. Neural network for the estimation of UV erythemal irradiance using solar broadband irradiance. International Journal of Climatology 27, 1791-1799. https://doi.org/10.1002/joc.1496

Alados, I., Mellado, J.A., Ramos, F., Alados-Arboledas, L., 2004. Estimating UV Erythemal Irradiance by Means of Neural NetworksII. phot 80, 351-358. https://doi.org/10.1562/2004-03-12-RA-111.1

Alfadda, A., Rahman, S., Pipattanasomporn, M., 2018. Solar irradiance forecast using aerosols measurements: A data driven approach. Solar Energy 170, 924-939. https://doi.org/10.1016/j.solener.2018.05.089

Allaart, M., Weele, M. van, Fortuin, P., Kelder, H., 2004. An empirical model to predict the UVindex based on solar zenith angles and total ozone. Meteorological Applications 11, 5965. https://doi.org/10.1017/S1350482703001130

Anderiesz, C., Elwood, M., Hill, D.J., 2006. Cancer control policy in Australia. Aust New Zealand Health Policy 3, 12. https://doi.org/10.1186/1743-8462-3-12

Antanasijević, D., Pocajt, V., Perić-Grujić, A., Ristić, M., 2014. Modelling of dissolved oxygen in the Danube River using artificial neural networks and Monte Carlo Simulation uncertainty analysis. Journal of Hydrology 519, 1895-1907. https://doi.org/10.1016/j.jhydrol.2014.10.009

ARPANSA, 2021. Australian Radiation and Nuclear Protection Agency 2021 Realtime UV levels [WWW Document]. ARPANSA. URL https://www.arpansa.gov.au/ourservices/monitoring/ultraviolet-radiation-monitoring/ultraviolet-radiation-index (accessed 7.9.21).

Barrett, P., Hunter, J., Miller, J.T., Hsu, J.-C., Greenfield, P., 2005. matplotlib -- A Portable Python Plotting Package 347, 91.

Baumgaertner, A.J.G., Seppälä, A., Jöckel, P., Clilverd, M.A., 2011. Geomagnetic activity related $\mathrm{NO}_{\mathrm{x}}$ enhancements and polar surface air temperature variability in a chemistry climate model: modulation of the NAM index. Atmospheric Chemistry and Physics 11, 4521-4531. https://doi.org/10.5194/acp-11-4521-2011

BOM, 2020. Australia's official weather forecasts \& weather radar - Bureau of Meteorology [WWW Document]. URL http://www.bom.gov.au/ (accessed 7.9.21).

Boniol, M., 2016. Descriptive epidemiology of skin cancer incidence and mortality, in: Skin Cancer Prevention. CRC Press, pp. 221-242.

Brownlee, J., 2016. Deep Learning With Python: Develop Deep Learning Models on Theano and TensorFlow Using Keras. Machine Learning Mastery.

Chai, T., Draxler, R.R., 2014. Root mean square error (RMSE) or mean absolute error (MAE)? Arguments against avoiding RMSE in the literature. Geoscientific Model Development 7, 1247-1250. https://doi.org/10.5194/gmd-7-1247-2014

Chen, C., Jiang, H., Zhang, Y., Wang, Y., 2010. Investigating spatial and temporal characteristics of harmful Algal Bloom areas in the East China Sea using a fast and flexible method, in: 2010 18th International Conference on Geoinformatics. Presented at the 2010 18th International Conference on Geoinformatics, pp. 1-4. https://doi.org/10.1109/GEOINFORMATICS.2010.5567490

Chen, J.P., Yang, L., Wang, L.K., Zhang, B., 2006. Ultraviolet Radiation for Disinfection, in: Wang, L.K., Hung, Y.-T., Shammas, N.K. (Eds.), Advanced Physicochemical Treatment Processes, Handbook of Environmental Engineering. Humana Press, Totowa, NJ, pp. 317-366. https://doi.org/10.1007/978-1-59745-029-4_10 
Deo, R.C., Downs, N., Parisi, A.V., Adamowski, J.F., Quilty, J.M., 2017. Very short-term reactive forecasting of the solar ultraviolet index using an extreme learning machine integrated with the solar zenith angle. Environmental Research 155, 141-166. https://doi.org/10.1016/j.envres.2017.01.035

Dey, R., Salem, F.M., 2017. Gate-variants of Gated Recurrent Unit (GRU) neural networks, in: 2017 IEEE 60th International Midwest Symposium on Circuits and Systems (MWSCAS). Presented at the 2017 IEEE 60th International Midwest Symposium on Circuits and Systems (MWSCAS), pp. 1597-1600. https://doi.org/10.1109/MWSCAS.2017.8053243

Downs, N., Butler, H., Parisi, A., 2016. Solar Ultraviolet Attenuation during the Australian (Red Dawn) Dust Event of 23 September 2009. Bulletin of the American Meteorological Society 97, 2039-2050. https://doi.org/10.1175/BAMS-D-15-00053.1

Eberhart, Shi, Y., 2001. Particle swarm optimization: developments, applications and resources, in: Proceedings of the 2001 Congress on Evolutionary Computation (IEEE Cat. No.01TH8546). Presented at the Proceedings of the 2001 Congress on Evolutionary Computation (IEEE Cat. No.01TH8546), pp. 81-86 vol. 1. https://doi.org/10.1109/CEC.2001.934374

Fan, J., Wu, L., Ma, X., Zhou, H., Zhang, F., 2020. Hybrid support vector machines with heuristic algorithms for prediction of daily diffuse solar radiation in air-polluted regions. Renewable Energy 145, 2034-2045. https://doi.org/10.1016/j.renene.2019.07.104

Fernández-Delgado, M., Cernadas, E., Barro, S., Ribeiro, J., Neves, J., 2014. Direct Kernel Perceptron (DKP): Ultra-fast kernel ELM-based classification with non-iterative closedform weight calculation. Neural Networks 50, 60-71. https://doi.org/10.1016/j.neunet.2013.11.002

Fouilloy, A., Voyant, C., Notton, G., Motte, F., Paoli, C., Nivet, M.-L., Guillot, E., Duchaud, J.L., 2018. Solar irradiation prediction with machine learning: Forecasting models selection method depending on weather variability. Energy 165, 620-629. https://doi.org/10.1016/j.energy.2018.09.116

Furuhashi, T., Torii, K., Ikumi, K., Kato, H., Nishida, E., Morita, A., 2020. Ultraviolet A1 phototherapy for the treatment of localized scleroderma. The Journal of Dermatology 47, 792-795. https://doi.org/10.1111/1346-8138.15368

Giovanni [WWW Document], 2021. URL https://giovanni.gsfc.nasa.gov/giovanni/ (accessed 7.9.21).

Gray, N.F., 2014. Chapter Thirty-Four - Ultraviolet Disinfection, in: Percival, S.L., Yates, M.V., Williams, D.W., Chalmers, R.M., Gray, N.F. (Eds.), Microbiology of Waterborne Diseases (Second Edition). Academic Press, London, pp. 617-630. https://doi.org/10.1016/B978-0-12-415846-7.00034-2

Guo, Y., Liu, Y., Oerlemans, A., Lao, S., Wu, S., Lew, M.S., 2016. Deep learning for visual understanding: A review. Neurocomputing, Recent Developments on Deep Big Vision 187, 27-48. https://doi.org/10.1016/j.neucom.2015.09.116

Hassan, R., Cohanim, B., Weck, O. de, Venter, G., 2004. A Comparison of Particle Swarm Optimization and the Genetic Algorithm, in: 46th AIAA/ASME/ASCE/AHS/ASC Structures, Structural Dynamics and Materials Conference. American Institute of Aeronautics and Astronautics. https://doi.org/10.2514/6.2005-1897

Heilingloh, C.S., Aufderhorst, U.W., Schipper, L., Dittmer, U., Witzke, O., Yang, D., Zheng, X., Sutter, K., Trilling, M., Alt, M., Steinmann, E., Krawczyk, A., 2020. Susceptibility of 
SARS-CoV-2 to UV irradiation. American Journal of Infection Control 48, 1273-1275. https://doi.org/10.1016/j.ajic.2020.07.031

Hendon, H., Salby, M., 1994. The Life Cycle of the Madden-Julian Oscillation. https://doi.org/10.1175/1520-0469(1994)051<2225:TLCOTM>2.0.CO;2

Hochreiter, S., Schmidhuber, J., 1997. Long Short-Term Memory. Neural Computation 9, 17351780. https://doi.org/10.1162/neco.1997.9.8.1735

Hollaender, A., Buy, H.G.D., Ingraham, H.S., Wheeler, S.M., 1944. Control of Air-Borne Microorganisms by Ultraviolet Floor Irradiation. Science 99, 130-131. https://doi.org/10.1126/science.99.2563.130

Huang, X., Zhang, C., Li, Q., Tai, Y., Gao, B., Shi, J., 2020. A Comparison of Hour-Ahead Solar Irradiance Forecasting Models Based on LSTM Network [WWW Document]. Mathematical Problems in Engineering. https://doi.org/10.1155/2020/4251517

Igoe, D., Parisi, A., Carter, B., 2013a. Smartphones as tools for delivering sun-smart education to students. Teaching Science 59, 36-38.

Igoe, D., Parisi, A., Carter, B., 2013b. Characterization of a smartphone camera's response to ultraviolet A radiation. Photochem Photobiol 89, 215-218. https://doi.org/10.1111/j.1751-1097.2012.01216.x

Isah, O.R., Usman, A.D., Tekanyi, A.M.S., 2017. A Hybrid Model of PSO Algorithm and Artificial Neural Network for Automatic Follicle Classification 16.

Ji, X., Shang, X., Dahlgren, R.A., Zhang, M., 2017. Prediction of dissolved oxygen concentration in hypoxic river systems using support vector machine: a case study of Wen-Rui Tang River, China. Environmental Science and Pollution Research 24, 16062 16076. https://doi.org/10.1007/s11356-017-9243-7

Jiménez-Pérez, P.F., Mora-López, L., 2016. Modeling and forecasting hourly global solar radiation using clustering and classification techniques. Solar Energy 135, 682-691. https://doi.org/10.1016/j.solener.2016.06.039

Jovanovic, B., Collins, D., Braganza, K., Jakob, D., Jones, D.A., 2011. A high-quality monthly total cloud amount dataset for Australia. Climatic Change 108, 485-517. https://doi.org/10.1007/s10584-010-9992-5

Juzeniene, A., Moan, J., 2012. Beneficial effects of UV radiation other than via vitamin D production. Dermato-Endocrinology 4, 109-117. https://doi.org/10.4161/derm.20013

Kaba, K., Kandirmaz, H.M., Avci, M., 2017. Estimation of daily sunshine duration using support vector machines. International Journal of Green Energy 14, 430-441. https://doi.org/10.1080/15435075.2016.1265971

Karimkhani, C., Green, A.C., Nijsten, T., Weinstock, M.A., Dellavalle, R.P., Naghavi, M., Fitzmaurice, C., 2017. The global burden of melanoma: results from the Global Burden of Disease Study 2015. British Journal of Dermatology 177, 134-140. https://doi.org/10.1111/bjd.15510

Kazantzidis, A., Smedley, A., Kift, R., Rimmer, J., Berry, J.L., Rhodes, L.E., Webb, A.R., 2015. A modeling approach to determine how much UV radiation is available across the UK and Ireland for health risk and benefit studies. Photochem. Photobiol. Sci. 14, 10731081. https://doi.org/10.1039/C5PP00008D

Kennedy, J., Eberhart, R., 1995. Particle swarm optimization, in: Proceedings of ICNN'95 International Conference on Neural Networks. Presented at the Proceedings of ICNN'95 International Conference on Neural Networks, pp. 1942-1948 vol.4. https://doi.org/10.1109/ICNN.1995.488968 
931

932

933

934

935

936

937

938

939

940

941

942

943

944

945

946

947

948

949

950

951

952

953

954

955

956

957

958

959

960

961

962

963

964

965

966

967

968

969

970

971

972

973

974

975
Ketkar, N., 2017. Introduction to Keras, in: Ketkar, N. (Ed.), Deep Learning with Python: A Hands-on Introduction. Apress, Berkeley, CA, pp. 97-111. https://doi.org/10.1007/978-14842-2766-4_7

Kiladis, G.N., Straub, K.H., Reid, G.C., Gage, K.S., 2001. Aspects of interannual and intraseasonal variability of the tropopause and lower stratosphere. Quarterly Journal of the Royal Meteorological Society 127, 1961-1983. https://doi.org/10.1002/qj.49712757606

Kroft, E.B.M., Berkhof, N.J.G., Kerkhof, P.C.M. van de, Gerritsen, R.M.J.P., Jong, E.M.G.J. de, 2008. Ultraviolet A phototherapy for sclerotic skin diseases: A systematic review. Journal of the American Academy of Dermatology 59, 1017-1030. https://doi.org/10.1016/j.jaad.2008.07.042

Krzyścin, J.W., Guzikowski, J., Czerwińska, A., Lesiak, A., Narbutt, J., Jarosławski, J., Sobolewski, P.S., Rajewska-Więch, B., Wink, J., 2015. 24 hour forecast of the surface UV for the antipsoriatic heliotherapy in Poland. Journal of Photochemistry and Photobiology B: Biology 148, 136-144. https://doi.org/10.1016/j.jphotobiol.2015.04.002

Latosińska, J.N., Latosińska, M., Bielak, J., 2015. Towards modelling ultraviolet index in global scale. Artificial neural networks approach. Aerospace Science and Technology 41, 189198. https://doi.org/10.1016/j.ast.2014.12.013

Lau, W.K.-M., Waliser, D.E., 2011. Intraseasonal variability in the atmosphere-ocean climate system. Springer Science \& Business Media.

Lecun, Y., Bottou, L., Bengio, Y., Haffner, P., 1998. Gradient-based learning applied to document recognition. Proceedings of the IEEE 86, 2278-2324. https://doi.org/10.1109/5.726791

Lee, S.-W., Hwang, S.-J., Lee, S.-B., Hwang, H.-S., Sung, H.-C., 2009. Landscape ecological approach to the relationships of land use patterns in watersheds to water quality characteristics. Landscape and Urban Planning 92, 80-89. https://doi.org/10.1016/J.LANDURBPLAN.2009.02.008

Legates, D.R., McCabe, G.J., 2013. A refined index of model performance: a rejoinder. International Journal of Climatology 33, 1053-1056. https://doi.org/10.1002/joc.3487

Li, J., Jiang, Y., Xia, X., Hu, Y., 2018. Increase of surface solar irradiance across East China related to changes in aerosol properties during the past decade. Environ. Res. Lett. 13, 034006. https://doi.org/10.1088/1748-9326/aaa35a

Li, Youxing., Chen, X., Yu, X., 2019. Processes | Free Full-Text | A Hybrid Energy Feature Extraction Approach for Ship-Radiated Noise Based on CEEMDAN Combined with Energy Difference and Energy Entropy [WWW Document]. URL https://www.mdpi.com/2227-9717/7/2/69 (accessed 6.19.21).

Liu, B., Wang, D., Fu, S., Cao, W., 2017. Estimation of Peak Flow Rates for Small Drainage Areas. Water Resources Management 31, 1635-1647. https://doi.org/10.1007/s11269017-1604-y

Liu, H., Tian, H., Li, Y., 2015. Four wind speed multi-step forecasting models using extreme learning machines and signal decomposing algorithms. Energy Conversion and Management 100, 16-22. https://doi.org/10.1016/j.enconman.2015.04.057

Lucas, R.M., McMichael, A.J., Armstrong, B.K., Smith, W.T., 2008. Estimating the global disease burden due to ultraviolet radiation exposure. International Journal of Epidemiology 37, 654-667. https://doi.org/10.1093/ije/dyn017 
976

977

978

979

980

981

982

983

984

985

986

987

988

989

990

991

992

993

994

995

996

997

998

999

1000

1001

1002

1003

1004

1005

1006

1007

1008

1009

1010

1011

1012

1013

1014

1015

1016

1017

1018

1019

1020

1021

Madden, R.A., Julian, P.R., 1994. Observations of the 40-50-Day Tropical Oscillation-A Review. Monthly Weather Review 122, 814-837. https://doi.org/10.1175/15200493(1994)122<0814:OOTDTO >2.0.CO;2

Madden, R.A., Julian, P.R., 1971. Detection of a 40-50 Day Oscillation in the Zonal Wind in the Tropical Pacific. Journal of the Atmospheric Sciences 28, 702-708. https://doi.org/10.1175/1520-0469(1971)028<0702:DOADOI >2.0.CO;2

Mäusezahl, D., Christen, A., Pacheco, G.D., Tellez, F.A., Iriarte, M., Zapata, M.E., Cevallos, M., Hattendorf, J., Cattaneo, M.D., Arnold, B., Smith, T.A., Colford, J.M., 2009. Solar drinking water disinfection (SODIS) to reduce childhood diarrhoea in rural Bolivia: a cluster-randomized, controlled trial. PLoS Med 6, e1000125. https://doi.org/10.1371/journal.pmed.1000125

Mayer, A., Lobet, M., 2018. UV to near-infrared broadband pyramidal absorbers via a genetic algorithm optimization approach, in: Metamaterials XI. Presented at the Metamaterials XI, International Society for Optics and Photonics, p. 1067127. https://doi.org/10.1117/12.2303823

McCarthy, W.H., 2004. The Australian experience in sun protection and screening for melanoma. J Surg Oncol 86, 236-245. https://doi.org/10.1002/jso.20086

Mucherino, A., Fidanova, S., Ganzha, M., 2015. Ant Colony Optimization with environment changes: an application to GPS surveying. Presented at the 2015 Federated Conference on Computer Science and Information Systems, pp. 495-500. https://doi.org/10.15439/2015F33

Nash, J.E., Sutcliffe, J.V., 1970. River flow forecasting through conceptual models part I - A discussion of principles. Journal of Hydrology 10, 282-290. https://doi.org/10.1016/0022-1694(70)90255-6

Norval, M., Cullen, A.P., de Gruijl, F.R., Longstreth, J., Takizawa, Y., Lucas, R.M., Noonan, F.P., van der Leun, J.C., 2007. The effects on human health from stratospheric ozone depletion and its interactions with climate change. Photochem Photobiol Sci 6, 232-251. https://doi.org/10.1039/b700018a

Opländer Christian, Volkmar Christine M., Paunel-Görgülü Adnana, van Faassen Ernst E., Heiss Christian, Kelm Malte, Halmer Daniel, Mürtz Manfred, Pallua Norbert, Suschek Christoph V., 2009. Whole Body UVA Irradiation Lowers Systemic Blood Pressure by Release of Nitric Oxide From Intracutaneous Photolabile Nitric Oxide Derivates. Circulation Research 105, 1031-1040. https://doi.org/10.1161/CIRCRESAHA.109.207019

Pak, U., Kim, C., Ryu, U., Sok, K., Pak, S., 2018. A hybrid model based on convolutional neural networks and long short-term memory for ozone concentration prediction. Air Qual Atmos Health 11, 883-895. https://doi.org/10.1007/s11869-018-0585-1

Parisi, A.V., Downs, N., Turner, J., Amar, A., 2016. Online educative activities for solar ultraviolet radiation based on measurements of cloud amount and solar exposures. Journal of Photochemistry and Photobiology B: Biology 162, 434-440. https://doi.org/10.1016/j.jphotobiol.2016.07.015

Pavlakis, K.G., Hatzianastassiou, N., Matsoukas, C., Fotiadi, A., Vardavas, I., 2008. ENSO surface shortwave radiation forcing over the tropical Pacific. Atmospheric Chemistry and Physics 8, 5565-5577. https://doi.org/10.5194/acp-8-5565-2008

Pavlakis, K.G., Hatzidimitriou, D., Drakakis, E., Matsoukas, C., Fotiadi, A., Hatzianastassiou, N., Vardavas, I., 2007. ENSO surface longwave radiation forcing over the tropical 
1022

1023

1024

1025

1026

1027

1028

1029

1030

1031

1032

1033

1034

1035

1036

1037

1038

1039

1040

1041

1042

1043

1044

1045

1046

1047

1048

1049

1050

1051

1052

1053

1054

1055

1056

1057

1058

1059

1060

1061

1062

1063

1064

1065

1066
Pacific. Atmospheric Chemistry and Physics 7, 2013-2026. https://doi.org/10.5194/acp7-2013-2007

Peng, H., Ying, C., Tan, S., Hu, B., Sun, Z., 2018. An Improved Feature Selection Algorithm Based on Ant Colony Optimization. IEEE Access 6, 69203-69209. https://doi.org/10.1109/ACCESS.2018.2879583

Pinker, R.T., Grodsky, S., Zhang, B., Chen, W., 2017. ENSO Impact on Radiative Fluxes as Observed from Space. Journal of Geophysical Research - Oceans 122. https://doi.org/10.1002/2017JC012900

Pooi, C.K., Ng, HY, 2018. Review of low-cost point-of-use water treatment systems for developing communities. npj Clean Water 1, 1-8. https://doi.org/10.1038/s41545-0180011-0

Prasad, R., Ali, M., Kwan, P., Khan, H., 2019. Designing a multi-stage multivariate empirical mode decomposition coupled with ant colony optimization and random forest model to forecast monthly solar radiation. Applied Energy 236, 778-792. https://doi.org/10.1016/j.apenergy.2018.12.034

Pruthi, D., Bhardwaj, R., 2021. Modeling air quality index using optimized neuronal networks inspired by swarms. Environmental Engineering Research 26. https://doi.org/10.4491/eer.2020.469

Qing, X., Niu, Y., 2018. Hourly day-ahead solar irradiance prediction using weather forecasts by LSTM. Energy 148, 461-468. https://doi.org/10.1016/j.energy.2018.01.177

Raksasat, R., Sri-iesaranusorn, P., Pemcharoen, J., Laiwarin, P., Buntoung, S., Janjai, S., Boontaveeyuwat, E., Asawanonda, P., Sriswasdi, S., Chuangsuwanich, E., 2021. Accurate surface ultraviolet radiation forecasting for clinical applications with deep neural network. Sci Rep 11, 5031. https://doi.org/10.1038/s41598-021-84396-2

Román, R., Antón, M., Valenzuela, A., Gil, J.E., Lyamani, H., Miguel, A.D., Olmo, F.J., Bilbao, J., Alados-Arboledas, L., 2013. Evaluation of the desert dust effects on global, direct and diffuse spectral ultraviolet irradiance. Tellus B: Chemical and Physical Meteorology 65, 19578. https://doi.org/10.3402/tellusb.v65i0.19578

Roshan, D.R., Koc, M., Abdallah, A., Martin-Pomares, L., Isaifan, R., Fountoukis, C., 2020. UV Index Forecasting under the Influence of Desert Dust: Evaluation against Surface and Satellite-Retrieved Data. Atmosphere 11, 96. https://doi.org/10.3390/atmos11010096

Saraiya, M., Glanz, K., Briss, P.A., Nichols, P., White, C., Das, D., Smith, S.J., Tannor, B., Hutchinson, A.B., Wilson, K.M., Gandhi, N., Lee, N.C., Rimer, B., Coates, R.C., Kerner, J.F., Hiatt, R.A., Buffler, P., Rochester, P., 2004. Interventions to prevent skin cancer by reducing exposure to ultraviolet radiation: a systematic review. Am J Prev Med 27, $422-$ 466. https://doi.org/10.1016/j.amepre.2004.08.009

Seme, S., Štumberger, G., 2011. A novel prediction algorithm for solar angles using solar radiation and Differential Evolution for dual-axis sun tracking purposes. Solar Energy 85, 2757-2770. https://doi.org/10.1016/j.solener.2011.08.031

Silva, C.A., Sousa, J.M.C., Runkler, T.A., Sá da Costa, J.M.G., 2009. Distributed supply chain management using ant colony optimization. European Journal of Operational Research 199, 349-358.

Sivamani, R.K., Crane, L.A., Dellavalle, R.P., 2009. The benefits and risks of ultraviolet (UV) tanning and its alternatives: the role of prudent sun exposure. Dermatol Clin 27, 149-vi. https://doi.org/10.1016/j.det.2008.11.008 
1067

1068

1069

1070

1071

1072

1073

1074

1075

1076

1077

1078

1079

1080

1081

1082

1083

1084

1085

1086

1087

1088

1089

1090

1091

1092

1093

1094

1095

1096

1097

1098

1099

1100

1101

1102

1103

1104

1105

1106

1107

1108

1109

1110

Slevin, T., Clarkson, J., English, D., 2000. Skin Cancer Control Western Australia: Is it Working and What Have we Learned? Radiat. Prot. Dosim. 91. https://doi.org/10.1093/oxfordjournals.rpd.a033225

Srivastava, R., Tiwari, A.N., Giri, V.K., 2019. Solar radiation forecasting using MARS, CART, M5, and random forest model: A case study for India. Heliyon 5, e02692. https://doi.org/10.1016/j.heliyon.2019.e02692

Staiger, H., den Outer, P.N., Bais, A.F., Feister, U., Johnsen, B., Vuilleumier, L., 2008. Hourly resolved cloud modification factors in the ultraviolet. Atmospheric Chemistry and Physics 8, 2493-2508. https://doi.org/10.5194/acp-8-2493-2008

Stanton, W.R., Janda, M., Baade, P.D., Anderson, P., 2004. Primary prevention of skin cancer: a review of sun protection in Australia and internationally. Health Promot Int 19, 369-378. https://doi.org/10.1093/heapro/dah310

Staples, M., Marks, R., Giles, G., 1998. Trends in the incidence of non-melanocytic skin cancer (NMSC) treated in Australia 1985-1995: are primary prevention programs starting to have an effect? Int J Cancer 78, 144-148. https://doi.org/10.1002/(sici)10970215(19981005)78:2<144::aid-ijc3>3.0.co;2-z

Staples, M.P., Elwood, M., Burton, R.C., Williams, J.L., Marks, R., Giles, G.G., 2006. Nonmelanoma skin cancer in Australia: the 2002 national survey and trends since 1985. Med J Aust 184, 6-10. https://doi.org/10.5694/j.1326-5377.2006.tb00086.x

Sudhibrabha, S., Harold Buchanan Exell, R., Sukawat, D., 2006. Ultraviolet forecasting in Thailand. ScienceAsia 32, 107. https://doi.org/10.2306/scienceasia15131874.2006.32.107

Szenicer, A., Fouhey, D.F., Munoz-Jaramillo, A., Wright, P.J., Thomas, R., Galvez, R., Jin, M., Cheung, M.C.M., 2019. A deep learning virtual instrument for monitoring extreme UV solar spectral irradiance. Science Advances 5, eaaw6548. https://doi.org/10.1126/sciadv.aaw6548

Tartaglione, N., Toniazzo, T., Orsolini, Y., Otterå, O.H., 2020. Impact of solar irradiance and geomagnetic activity on polar NOx, ozone and temperature in WACCM simulations. Journal of Atmospheric and Solar-Terrestrial Physics 209, 105398. https://doi.org/10.1016/j.jastp.2020.105398

Tian, B., Waliser, D.E., Kahn, R.A., Li, Q., Yung, Y.L., Tyranowski, T., Geogdzhayev, I.V., Mishchenko, M.I., Torres, O., Smirnov, A., 2008. Does the Madden-Julian Oscillation influence aerosol variability? Journal of Geophysical Research: Atmospheres 113. https://doi.org/10.1029/2007JD009372

Timmermann, L.F., Ritter, K., Hillebrandt, D., Küpper, T., 2015. Drinking water treatment with ultraviolet light for travelers - Evaluation of a mobile lightweight system. Travel Medicine and Infectious Disease 13, 466-474. https://doi.org/10.1016/j.tmaid.2015.10.005

Tiwari, M.K., Adamowski, J., 2013. Urban water demand forecasting and uncertainty assessment using ensemble wavelet-bootstrap-neural network models. Water Resources Research 49, 6486-6507. https://doi.org/10.1002/wrcr.20517

Tiwari, M.K., Chatterjee, C., 2010. A new wavelet-bootstrap-ANN hybrid model for daily discharge forecasting. Journal of Hydroinformatics 13, 500-519.

https://doi.org/10.2166/hydro.2010.142 
1111

1112

1113

1114

1115

1116

1117

1118

1119

1120

1121

1122

1123

1124

1125

1126

1127

1128

1129

1130

1131

1132

1133

1134

1135

1136

1137

1138

1139

1140

1141

1142

1143

1144

1145

1146

1147

1148

1149

Turner, E.C., Manners, J., Morcrette, C.J., O’Hagan, J.B., Smedley, A.R.D., 2017. Toward a New UV Index Diagnostic in the Met Office's Forecast Model. Journal of Advances in Modeling Earth Systems 9, 2654-2671. https://doi.org/10.1002/2017MS001050

Ventor, G., Sobieszczanski-Sobieski, J., 2003. Particle Swarm Optimization | AIAA Journal [WWW Document]. URL https://arc.aiaa.org/doi/10.2514/2.2111 (accessed 6.19.21).

Wang, F., Yu, Y., Zhang, Z., Li, J., Zhen, Z., Li, K., 2018. Wavelet Decomposition and Convolutional LSTM Networks Based Improved Deep Learning Model for Solar Irradiance Forecasting. Applied Sciences 8, 1286. https://doi.org/10.3390/app8081286

Waskom, M.L., 2021. seaborn: statistical data visualization. Journal of Open Source Software 6, 3021. https://doi.org/10.21105/joss.03021

Weile, D., Michielssen, E., 1997. Genetic algorithm optimization applied to electromagnetics: a review. https://doi.org/10.1109/8.558650

Welch, D., Buonanno, M., Grilj, V., Shuryak, I., Crickmore, C., Bigelow, A.W., RandersPehrson, G., Johnson, G.W., Brenner, D.J., 2018. Far-UVC light: A new tool to control the spread of airborne-mediated microbial diseases. Scientific Reports 8, 2752. https://doi.org/10.1038/s41598-018-21058-w

Wells, W.F., Fair, GM, 1935. Viability of B. Coli Exposed to Ultra-Violet Radiation in Air. Science 82, 280-281. https://doi.org/10.1126/science.82.2125.280-a

Wheeler, M.C., Hendon, H.H., 2004. An All-Season Real-Time Multivariate MJO Index: Development of an Index for Monitoring and Prediction. Monthly Weather Review 132, 1917-1932.

WHO, 2002. Global solar UV index: a practical guide : a joint recommendation of World Health Organization, World Meteorological Organization, United Nations Environment Programme, International Commission on Non-Ionizing Radiation Protection. Geneva, WHO.

Willmott, C.J., Robeson, S.M., Matsuura, K., 2012. A refined index of model performance. International Journal of Climatology 32, 2088-2094. https://doi.org/10.1002/joc.2419

Yadav, A.K., Chandel, SS, 2014. Solar radiation prediction using Artificial Neural Network techniques: A review. Renewable and Sustainable Energy Reviews 33, 772-781. https://doi.org/10.1016/j.rser.2013.08.055

Yan, H., Sun, L., Wang, Y., Huang, W., Qiu, S., Yang, C., 2011. A record of the Southern Oscillation Index for the past 2,000 years from precipitation proxies. Nature Geosci 4, 611-614. https://doi.org/10.1038/ngeo1231

Zhang, J., Zhang, X., Niu, J., Hu, B.X., Soltanian, M.R., Qiu, H., Yang, L., 2019. Prediction of groundwater level in seashore reclaimed land using wavelet and artificial neural networkbased hybrid model. Journal of Hydrology 577, 123948. https://doi.org/10.1016/j.jhydrol.2019.123948 
Table 1. Description of global pool of 24 predictor variables used to design and evaluate hybrid CEEMDAN-CNN-LSTM predictive model for the daily maximum UV Index forecasting.

\begin{tabular}{|c|c|c|}
\hline \multicolumn{3}{|c|}{ MODIS-Satellite } \\
\hline OTC & Ozone Total Column & DU \\
\hline GH & Geopotential Height (Daytime) & - \\
\hline $\mathrm{AO}$ & Aerosol Optical Depth $550 \mathrm{~nm}$ & - \\
\hline AOD2 & Aerosol Optical Depth $342.5 \mathrm{~nm}$ & - \\
\hline TCW & Total Column Water Vapour (Daytime) & $\mathrm{Kg} / \mathrm{m}^{2}$ \\
\hline $\mathrm{CF}$ & Cloud Fraction (Daytime) & - \\
\hline $\mathrm{CP}$ & Cloud Pressure (Daytime) & $\mathrm{hPa}$ \\
\hline $\mathrm{CCO}$ & Combined Cloud Optical Thickness (Mean) & - \\
\hline \multicolumn{3}{|c|}{ SILO (Ground- Based Observations) } \\
\hline T.Max & Maximum Temperature & ${ }^{\circ} \mathrm{C}$ \\
\hline T.Min & Minimum Temperature & ${ }^{\circ} \mathrm{C}$ \\
\hline Rain & Rainfall & $\mathrm{mm}$ \\
\hline Evap & Evaporation & $\mathrm{mm}$ \\
\hline Radn & Radiation & $\mathrm{MJ} \mathrm{m}^{-2}$ \\
\hline VP & Vapour Pressure & $\mathrm{hPa}$ \\
\hline RHmaxT & Relative Humidity at Temperature T.Max & $\%$ \\
\hline RHminT & Relative Humidity at Temperature T.Min & $\%$ \\
\hline FAO56 & Morton potential evapotranspiration overland & $\mathrm{mm}$ \\
\hline \multicolumn{3}{|c|}{ SYNOPTIC-SCALE (Climate Mode Indices) } \\
\hline Nino3.0 & Average SSTA over $150^{\circ}-90^{\circ} \mathrm{W}$ and $5^{\circ} \mathrm{N}-5^{\circ} \mathrm{S}$ & \multirow{15}{*}{$\begin{array}{l}\text { 号 } \\
0 \\
Z\end{array}$} \\
\hline Nino3.4 & Average SSTA over $170^{\circ} \mathrm{E}-120^{\circ} \mathrm{W}$ and $5^{\circ} \mathrm{N}-5^{\circ} \mathrm{S}$ & \\
\hline Nino4.0 & Average SSTA over $160^{\circ} \mathrm{E}-150^{\circ} \mathrm{W}$ and $5^{\circ} \mathrm{N}-5^{\circ} \mathrm{S}$ & \\
\hline Nino1+2 & Average SSTA over $90^{\circ} \mathrm{W}-80^{\circ} \mathrm{W}$ and $0^{\circ}-10^{\circ} \mathrm{S}$ & \\
\hline $\mathrm{AON}$ & Arctic Oscillation & \\
\hline $\mathrm{AAO}$ & Antarctic Oscillation & \\
\hline $\mathrm{EPO}$ & East Pacific Oscillation & \\
\hline GBI & Greenland Blocking Index (GBI) & \\
\hline WPO & Western Pacific Oscillation (WPO.) & \\
\hline PNA & Pacific North American Index & \\
\hline NAO & North Atlantic Oscillation & \\
\hline SAM & Southern Annular Mode index & \\
\hline SOI & Southern Oscillation Index, as per Troup (1965) & \\
\hline RMM1 & Real-Time Multivariate MJO indices 1 & \\
\hline RMM2 & Real-Time Multivariate MJO indices 1 & \\
\hline
\end{tabular}


Table 2. List of selected input variables prior applying in the proposed model using four optimization techniques (i.e., ACO, DEV, GA and PSO).

\begin{tabular}{lcccc}
\hline & ACO & DEV & GA & PSO \\
\hline OTC & $\checkmark$ & $\times$ & $\checkmark$ & $\times$ \\
\hline GH & $\times$ & $\checkmark$ & $\times$ & $\checkmark$ \\
\hline AOD & $\checkmark$ & $\checkmark$ & $\checkmark$ & $\times$ \\
\hline AOD2 & $\times$ & $\checkmark$ & $\times$ & $\times$ \\
\hline TCW & $\checkmark$ & $\times$ & $\checkmark$ & $\times$ \\
\hline CF & $\times$ & $\times$ & $\checkmark$ & $\checkmark$ \\
\hline CP & $\checkmark$ & $\times$ & $\times$ & $\times$ \\
\hline CCO & $\times$ & $\checkmark$ & $\times$ & $\checkmark$ \\
\hline T.Max & $\checkmark$ & $\times$ & $\times$ & $\times$ \\
\hline T.Min & $\times$ & $\times$ & $\times$ & $\checkmark$ \\
\hline Rain & $\times$ & $\checkmark$ & $\times$ & $\checkmark$ \\
\hline Evap & $\times$ & $\times$ & $\checkmark$ & $\times$ \\
\hline Radn & $\times$ & $\times$ & $\times$ & $\checkmark$ \\
\hline VP & $\times$ & $\times$ & $\times$ & $\times$ \\
\hline RHmaxT & $\times$ & $\times$ & $\times$ & $\times$ \\
\hline RHminT & $\times$ & $\times$ & $\checkmark$ & $\times$ \\
\hline FAO56 & $\times$ & $\times$ & $\times$ & $\times$ \\
\hline SOI & $\times$ & $\times$ & $\checkmark$ & $\times$ \\
\hline EPO & $\checkmark$ & $\times$ & $\times$ & $\times$ \\
\hline GBI & $\checkmark$ & $\times$ & $\checkmark$ & $\times$ \\
\hline AAO & $\times$ & $\times$ & $\checkmark$ & $\checkmark$ \\
\hline AO & $\checkmark$ & $\times$ & $\checkmark$ & $\times$ \\
\hline NAO & $\times$ & $\checkmark$ & $\checkmark$ & $\times$ \\
\hline PNA & $\checkmark$ & $\times$ & $\times$ & $\checkmark$ \\
\hline Nino3 & $\checkmark$ & $\times$ & $\times$ & $\times$ \\
\hline NINO4 & $\times$ & $\checkmark$ & $\checkmark$ & $\times$ \\
\hline NINO12 & $\times$ & $\checkmark$ & $\checkmark$ & $\times$ \\
\hline NINO34 & $\times$ & $\checkmark$ & $\times$ & $\times$ \\
\hline RMM2 & $\checkmark$ & $\checkmark$ & $\checkmark$ & $\checkmark$ \\
\hline
\end{tabular}


Table 3. Evaluation of hybrid CEEMDAN-CLSTM vs. benchmark (CNN-GRU, CNN-LSTM, CEEMDAN-GRU, CEEMDAN-LSTM, GRU and LSTM) models for Perth observation sites. The correlation coefficient ( $r$ ), root mean square error (RMSE), mean absolute error (MAE) and NashSutcliffe coefficient $(N S)$ are computed between forecasted and observed $U V I$ for 7 Day ahead periods in testing phase. The optimal model is

boldfaced (blue).

\begin{tabular}{|c|c|c|c|c|c|c|c|c|c|c|c|c|c|c|c|c|}
\hline & \multicolumn{4}{|c|}{$\mathrm{ACO}$} & \multicolumn{4}{|c|}{ DEV } & \multicolumn{4}{|c|}{ GA } & \multicolumn{4}{|c|}{ PSO } \\
\hline & $r$ & $N S$ & RMSE & $M A E$ & $r$ & $N S$ & $R M S E$ & $M A E$ & $r$ & $N S$ & $R M S E$ & $M A E$ & $r$ & $N S$ & $R M S E$ & $M A E$ \\
\hline CEEMDAN-CGRU & 0.995 & 0.991 & 0.317 & 0.261 & 0.994 & 0.981 & 0.460 & 0.392 & 0.994 & 0.994 & 0.260 & 0.198 & 0.995 & 0.990 & 0.343 & 0.281 \\
\hline CEEMDAN-CLSTM & 0.996 & 0.992 & 0.216 & 0.163 & 0.995 & 0.986 & 0.258 & 0.148 & 0.996 & 0.997 & 0.162 & 0.119 & 0.996 & 0.993 & 0.220 & 0.165 \\
\hline CEEMDAN-GRU & 0.973 & 0.945 & 0.796 & 0.598 & 0.993 & 0.987 & 0.387 & 0.300 & 0.974 & 0.948 & 0.768 & 0.579 & 0.993 & 0.985 & 0.413 & 0.312 \\
\hline CEEMDAN-LSTM & 0.982 & 0.963 & 0.648 & 0.483 & 0.983 & 0.967 & 0.615 & 0.447 & 0.992 & 0.981 & 0.463 & 0.354 & 0.978 & 0.949 & 0.766 & 0.563 \\
\hline CEEMDAN-DT & 0.960 & 0.920 & 0.952 & 0.672 & 0.986 & 0.971 & 0.575 & 0.420 & 0.959 & 0.918 & 0.968 & 0.685 & 0.984 & 0.968 & 0.599 & 0.425 \\
\hline CEEMDAN-MLP & 0.945 & 0.890 & 1.117 & 0.846 & 0.969 & 0.938 & 0.839 & 0.576 & 0.957 & 0.914 & 0.987 & 0.705 & 0.964 & 0.927 & 0.911 & 0.688 \\
\hline CEEMDAN-SVR & 0.988 & 0.974 & 0.541 & 0.384 & 0.993 & 0.987 & 0.387 & 0.300 & 0.990 & 0.978 & 0.504 & 0.370 & 0.991 & 0.991 & 0.385 & 0.223 \\
\hline CNN-GRU & 0.987 & 0.987 & 0.317 & 0.361 & 0.988 & 0.974 & 0.541 & 0.384 & 0.988 & 0.968 & 0.601 & 0.453 & 0.981 & 0.962 & 0.663 & 0.457 \\
\hline CNN-LSTM & 0.986 & 0.982 & 0.307 & 0.334 & 0.977 & 0.950 & 0.758 & 0.548 & 0.986 & 0.979 & 0.556 & 0.292 & 0.991 & 0.972 & 0.567 & 0.458 \\
\hline GRU & 0.973 & 0.945 & 0.796 & 0.598 & 0.959 & 0.913 & 0.986 & 0.767 & 0.968 & 0.933 & 0.875 & 0.644 & 0.975 & 0.948 & 0.771 & 0.559 \\
\hline LSTM & 0.977 & 0.950 & 0.758 & 0.548 & 0.980 & 0.954 & 0.721 & 0.508 & 0.975 & 0.945 & 0.793 & 0.537 & 0.981 & 0.958 & 0.690 & 0.485 \\
\hline DT & 0.884 & 0.766 & 1.631 & 1.112 & 0.878 & 0.753 & 1.675 & 1.161 & 0.877 & 0.752 & 1.678 & 1.148 & 0.950 & 0.897 & 1.082 & 0.752 \\
\hline MLP & 0.935 & 0.872 & 1.207 & 0.905 & 0.933 & 0.838 & 1.359 & 1.072 & 0.943 & 0.889 & 1.124 & 0.814 & 0.957 & 0.907 & 1.030 & 0.773 \\
\hline SVR & 0.957 & 0.914 & 0.990 & 0.655 & 0.939 & 0.878 & 1.178 & 0.809 & 0.948 & 0.896 & 1.090 & 0.733 & 0.970 & 0.940 & 0.826 & 0.557 \\
\hline
\end{tabular}



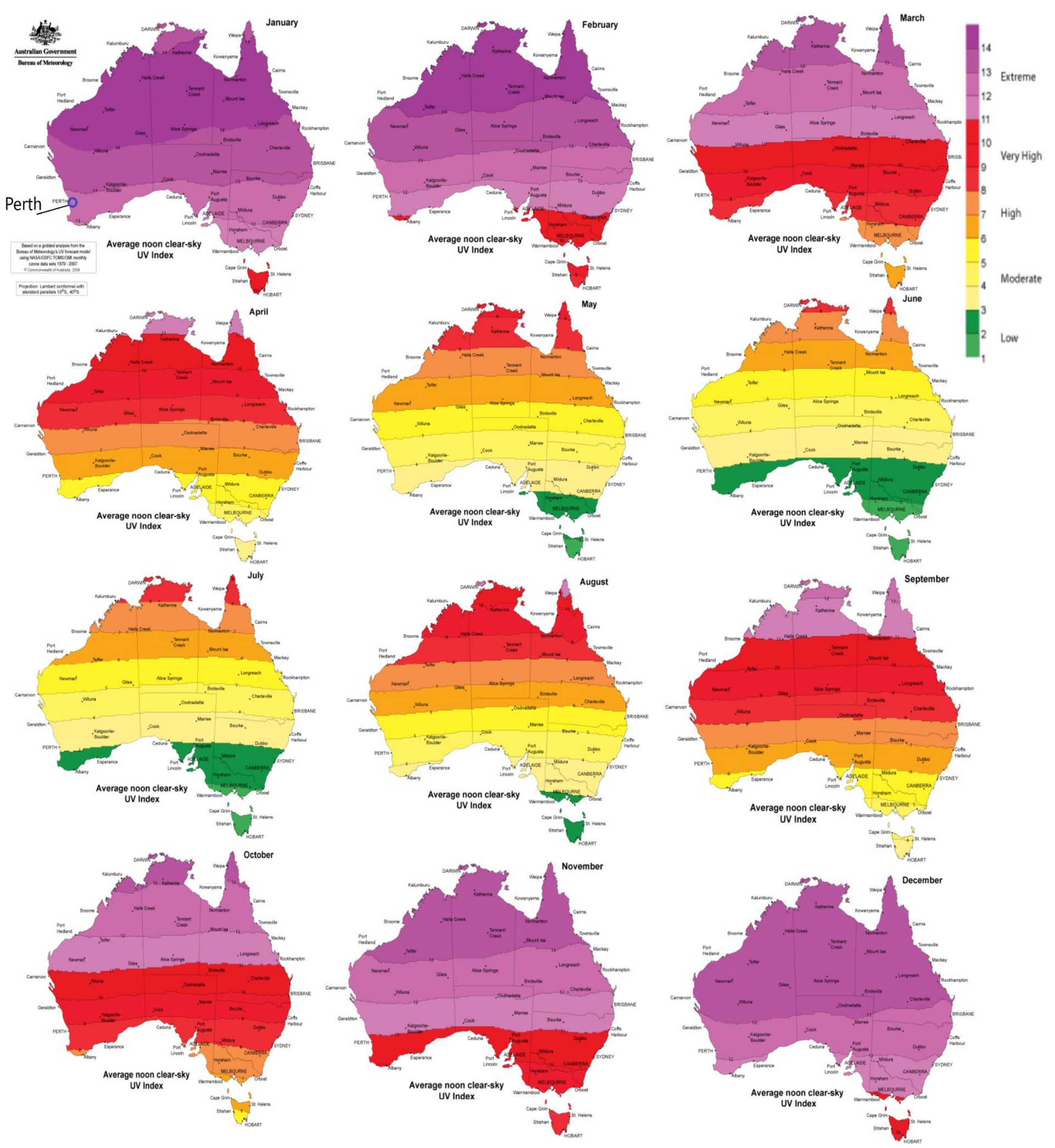

Figure 1 Study site (Perth, Australia) of the work and monthly average noon clear-sky UV index based on gridded analysis from the Bureau of Meteorology's UV forecast model using NASA/GFSC TOMS OMI monthly ozone data sets between 1979 - 2007 


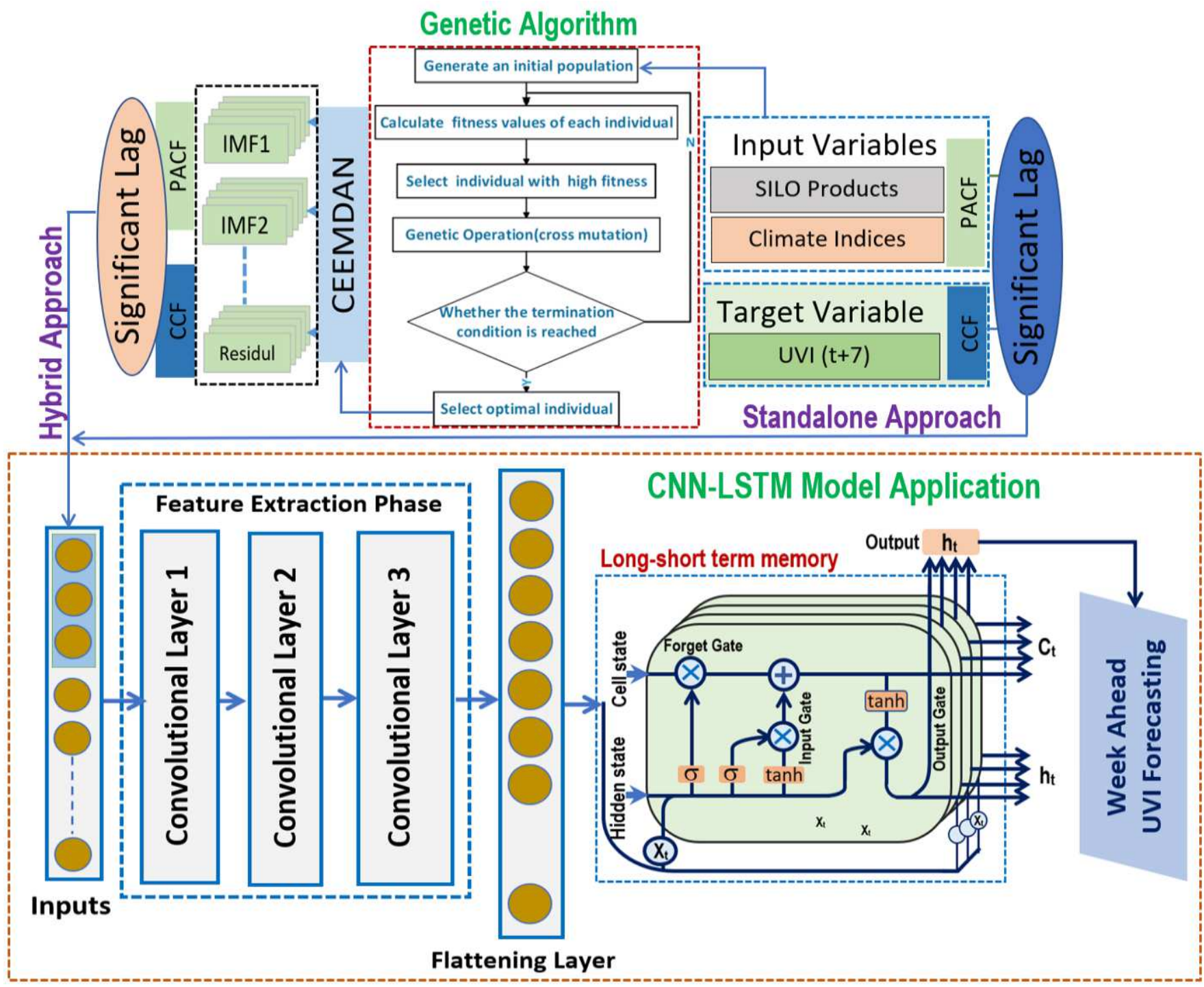

Figure 2 The developed model architecture of (Convolutional Neural Network, CNN) with the 4 layered long short term memory for a hybrid CNN-LSTM (CLSTM) model to forecast a week daily maximum UV Index with Genetic Algorithm. 

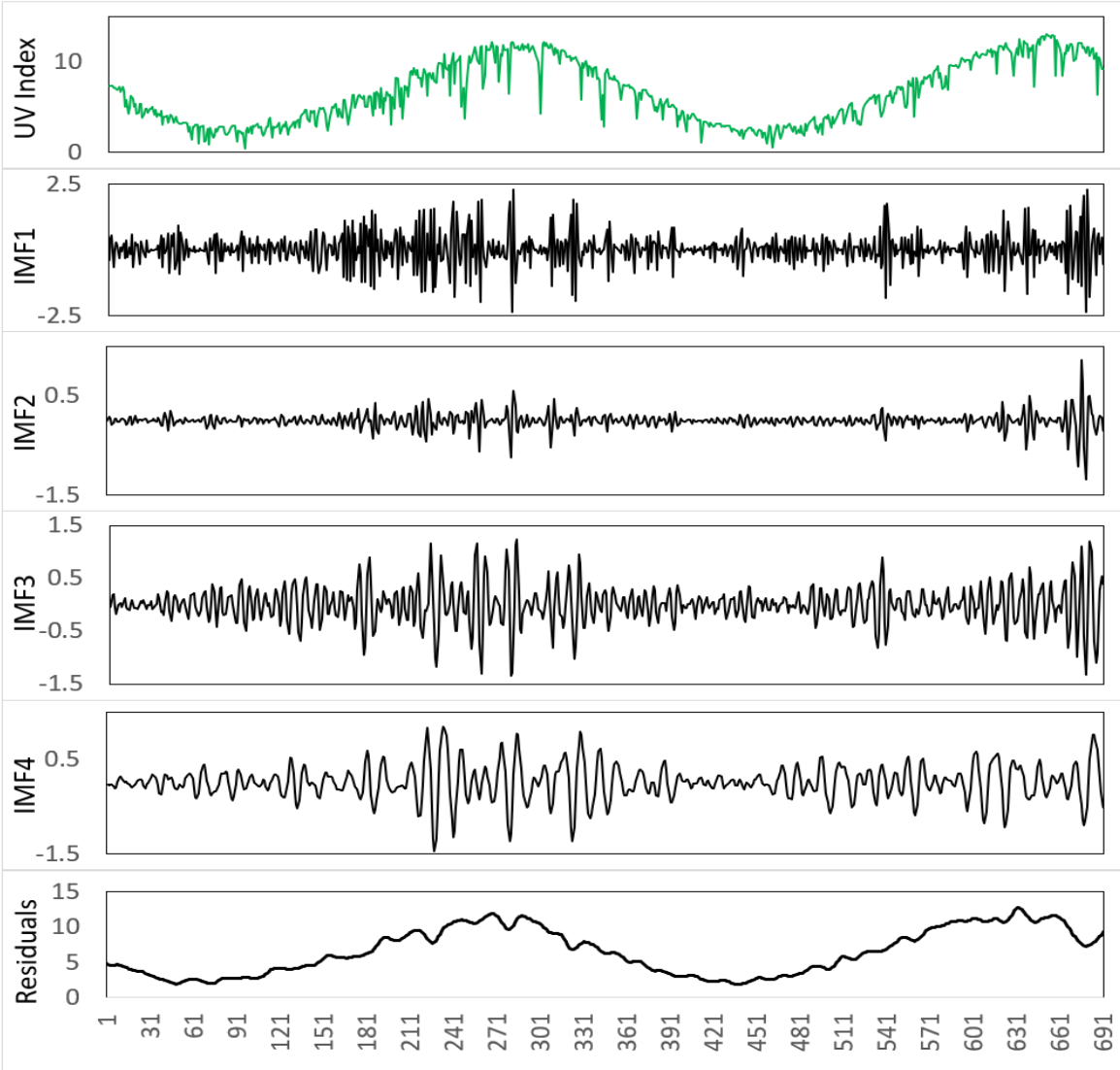

Figure 3 An example time-series showing data features in IMFs and residuals produced by the CEEMDAN transformation of daily maximum UV Index for the case of Perth study site. 

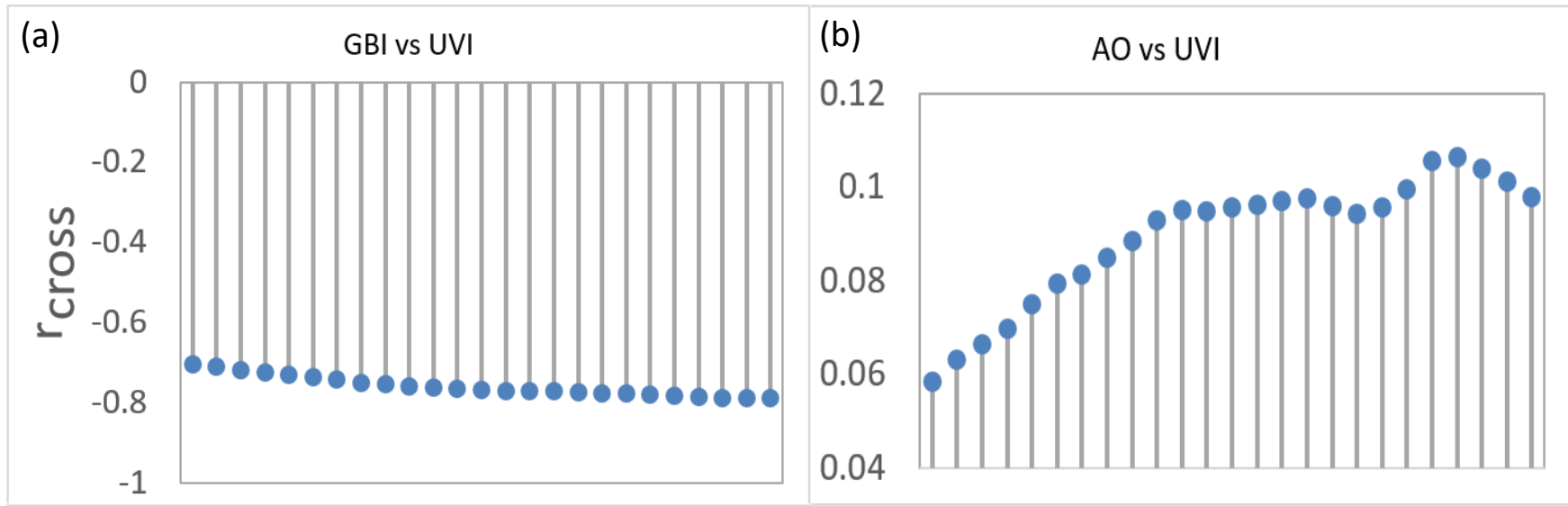

(c)

RMM2 vs UVI

(d)

T.Max Vs UVI

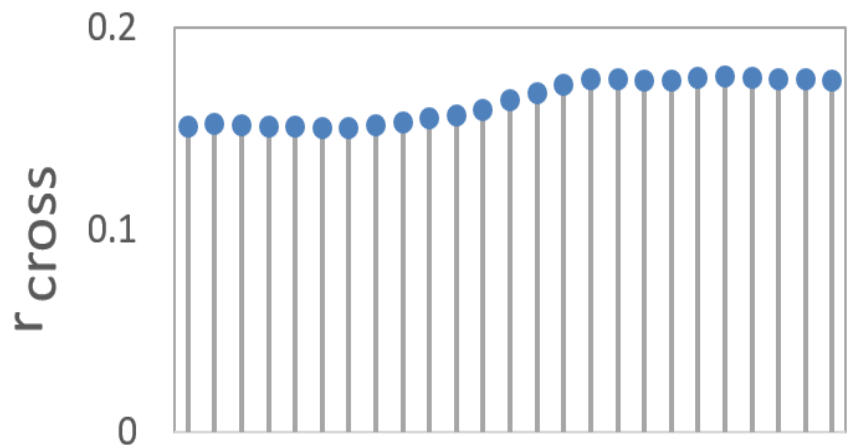

0.8

0.7

0.6

0.5

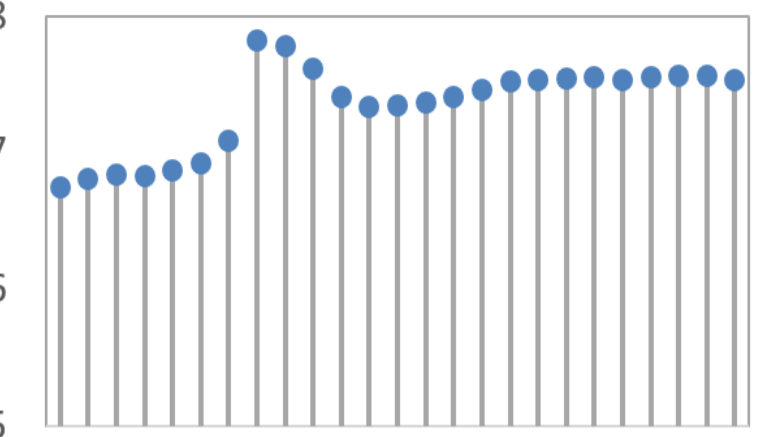

(e)

RMM2 vs UVI

(f)

UVI

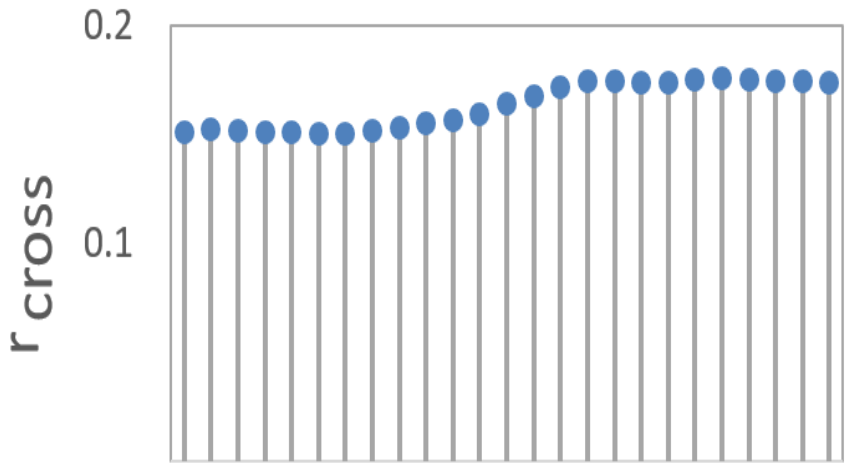

$01 \quad 3 \quad 5 \quad 7 \quad 911131517192123$ Lag (Days)

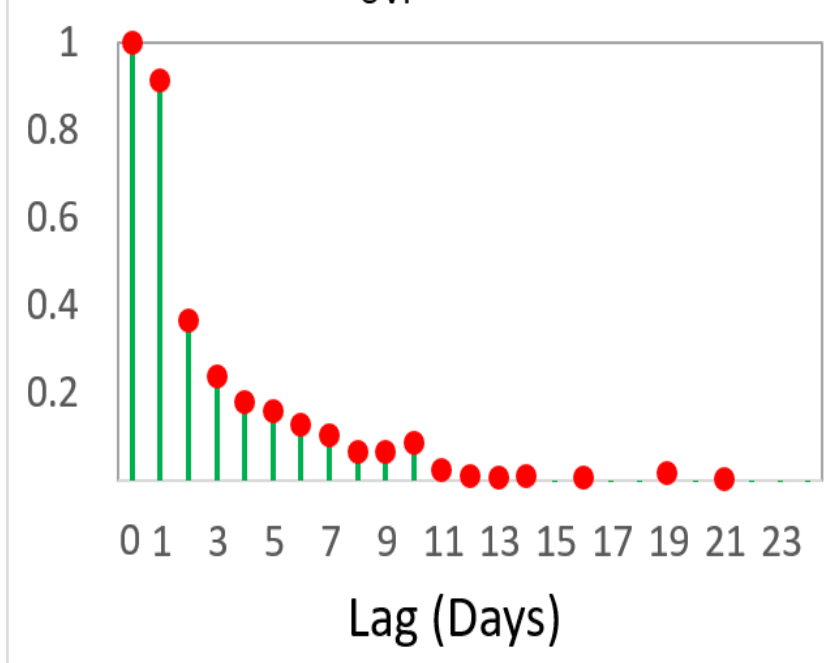

Figure 4 (a-e) Correlogram showing the covariance between the objective variable (UVI) and the predictor variables in terms of the Cross-correlation coefficient $\left(\mathrm{r}_{\text {cross }}\right)$ and (f) Partial autocorrelation function (PACF) plot of the UVI time series exploring the antecedent behavior in terms of the lag of UVI every day. 


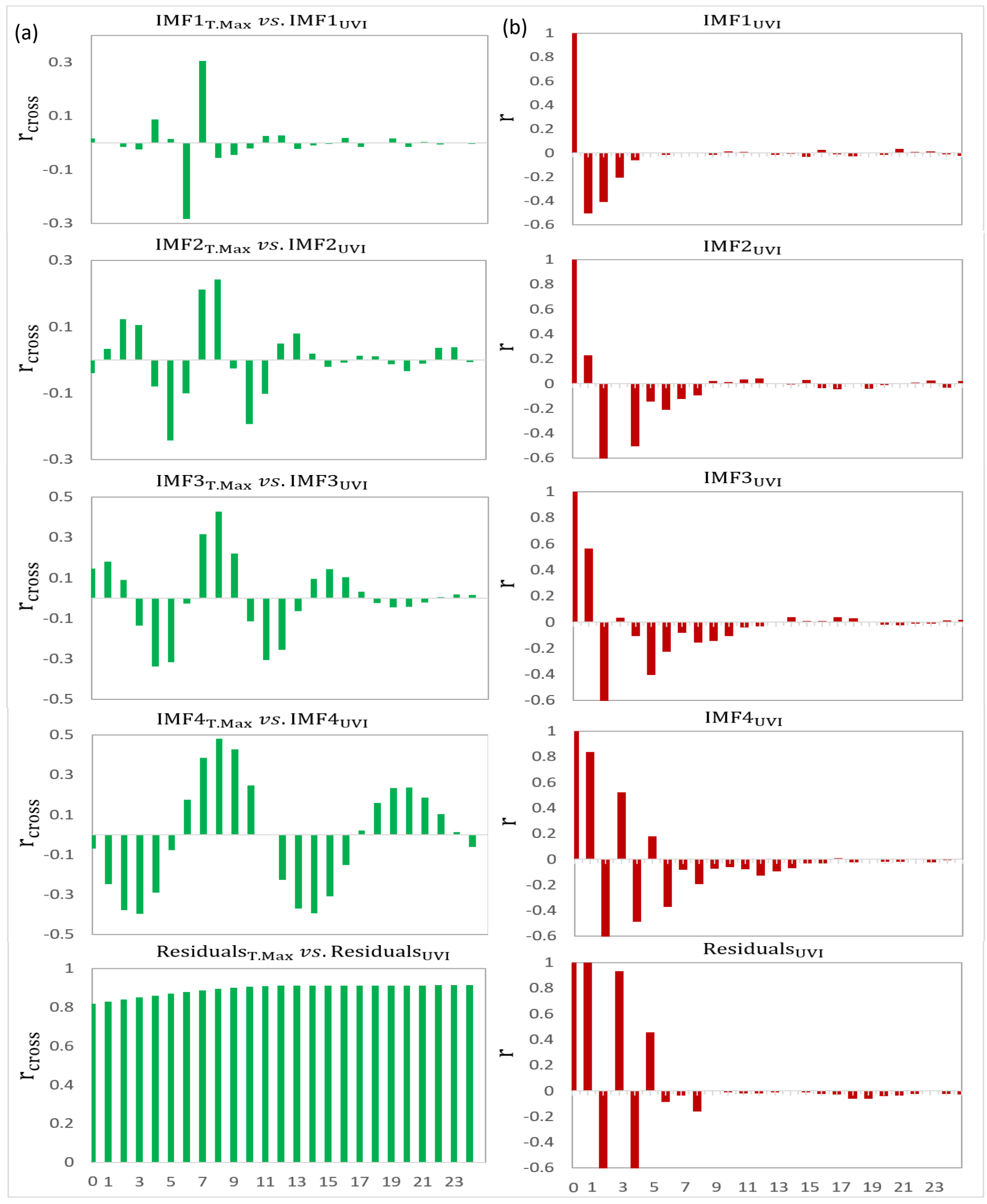

Figure 5 (a) Correlogram showing the covariance between the objective variable (UVI) and the CEEMDAN decomposed T.Max (IMF1 $1_{\text {T.Max }}$ to Residualst.Max) in terms of the Crosscorrelation coefficient $\left(\mathrm{r}_{\text {cross }}\right.$ ) and (b) Partial autocorrelation function (PACF) plot of the CEEMDAN decomposed UVI time series exploring the antecedent behavior in terms of the lag of UVI every day. 

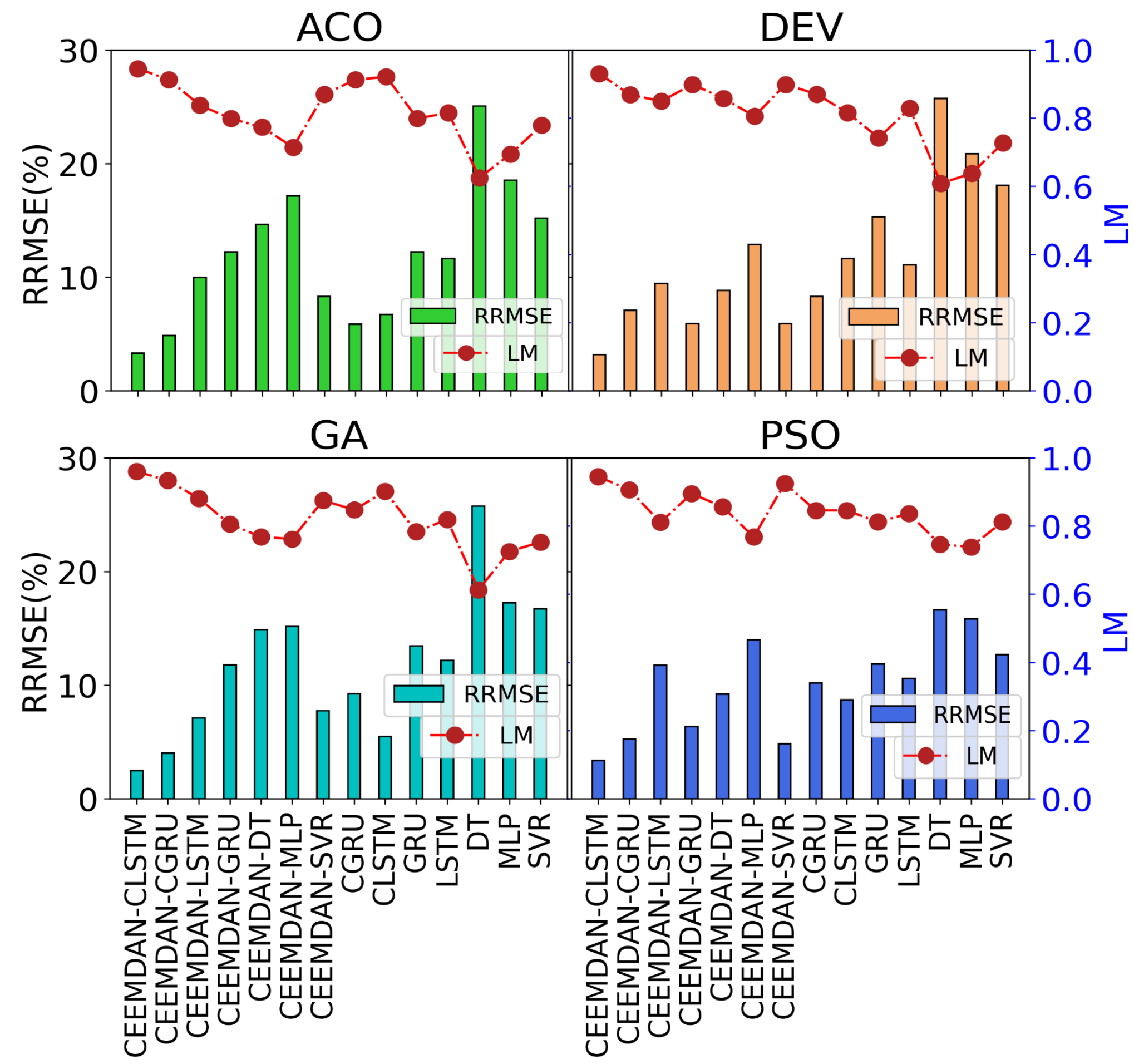

Figure 6 Comparison of the forecasting skill for all of the proposed models in terms of the relative error: RRMSE (\%) and Legate McCabe Index (LM) within the testing period. 


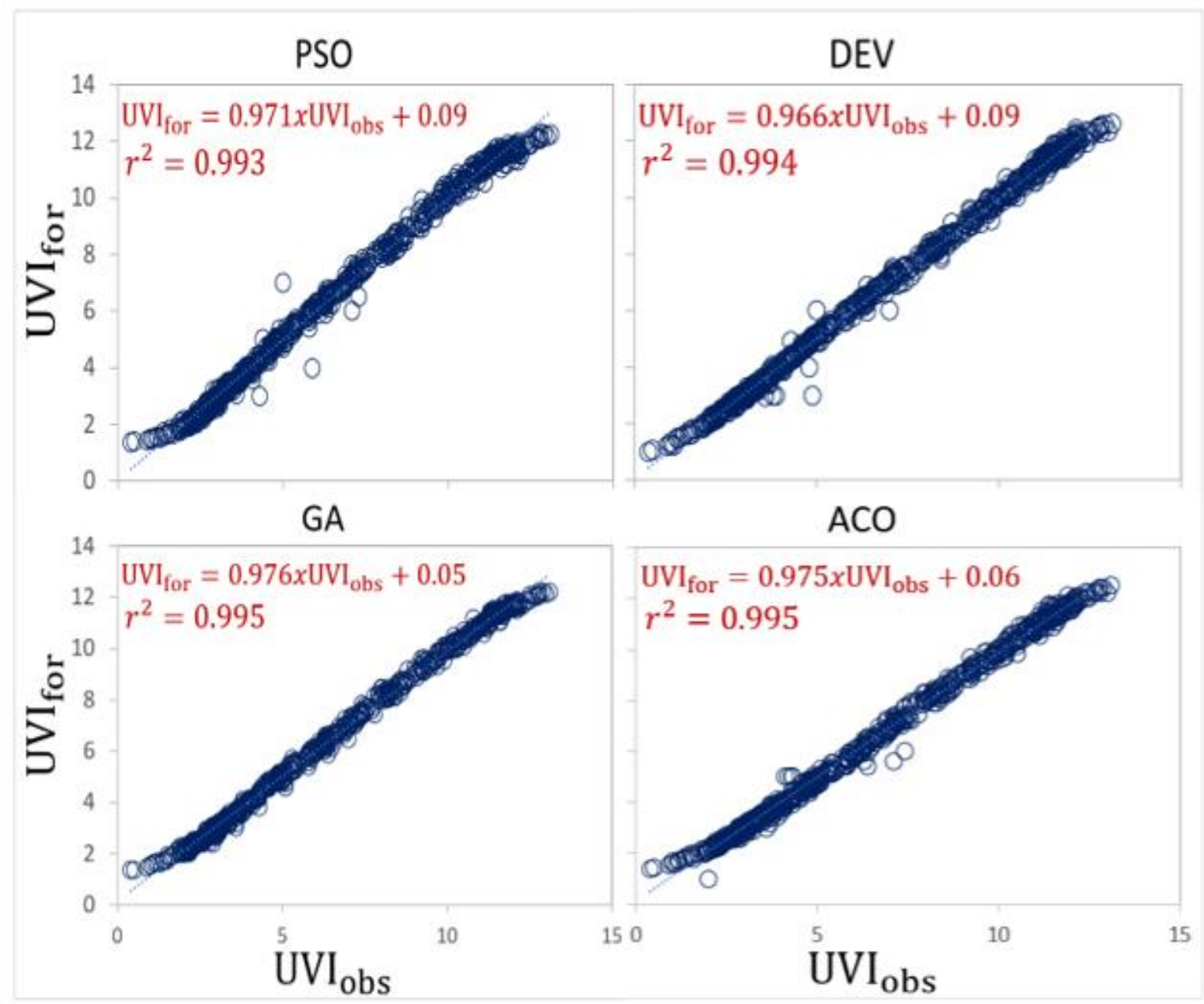

Figure 7 Scatter plot of forecasted with observed UVI (UVI) of Perth station CEEMDAN-CNN-GRU (CEEMDAN-CLSTM) model. A least square regression line and coefficient of determination $\left(\mathrm{R}^{2}\right)$ with a linear fit equation are shown in each sub-panel. 

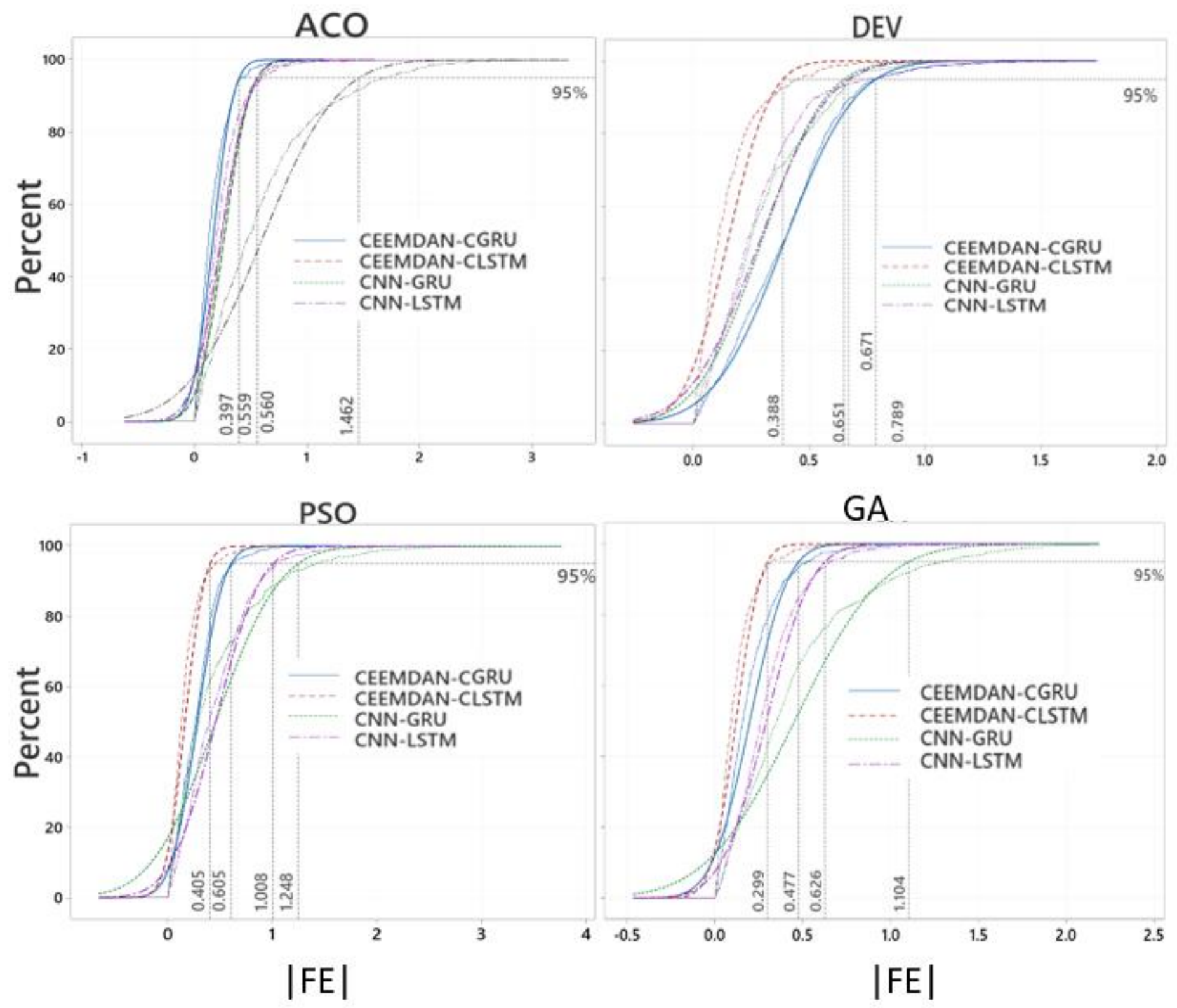

Figure 8 Empirical cumulative distribution function (CDF) in terms of forecasting error $|\mathrm{FE}|$ for CEEMDAN-CNN-GRU, CEEMDAN-CNN-LSTM, CNN-GRU, and CNN-LSTM model, shown for the 95 percentile on ECDF. 


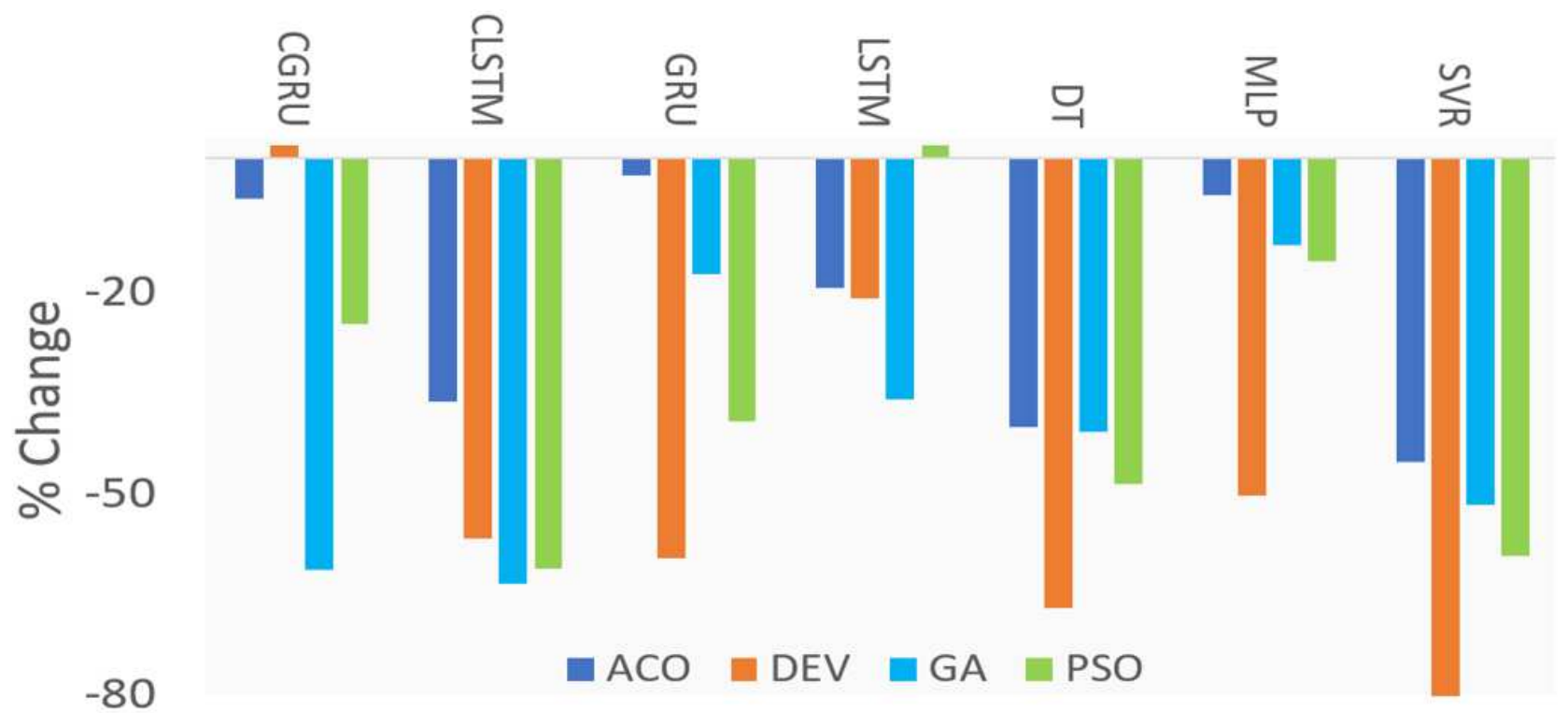

Figure 9 Effect on per cent change (\%) of RMAE using CEEMDAN as a feature extraction approach in forecasting UVI at Perth station using Genetic Algorithm (GA), Ant Colony Optimization (ACO), Particle Swarm Optimization (PSO), and Differential Evolution (DEV)
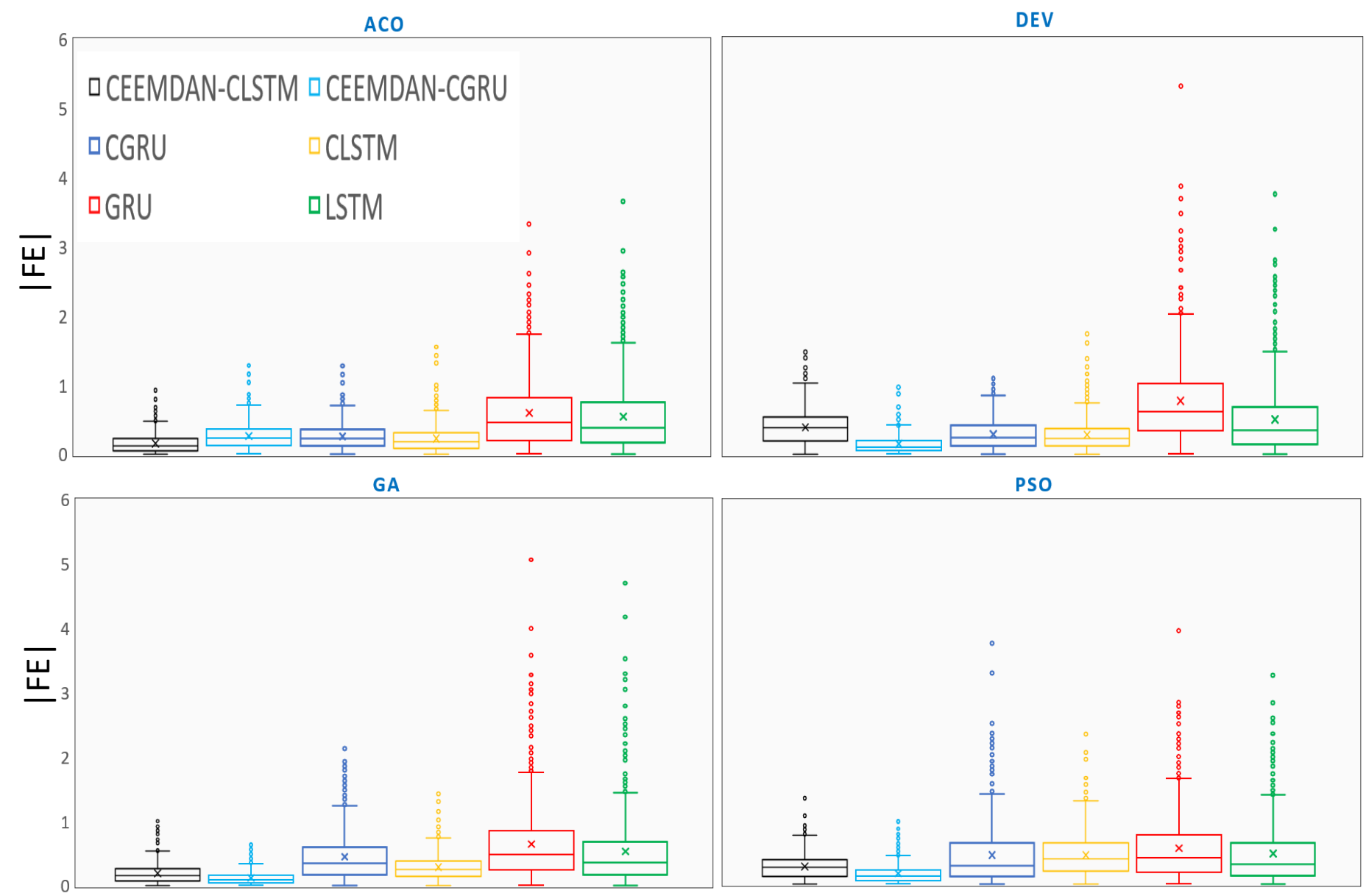

Figure 10 Evaluation of the performance of the proposed hybrid deep learning, CEEMDANCLSTM model with the comparative benchmark models based on the absolute forecasted error $|\mathrm{FE}|$ using four optimization techniques. 


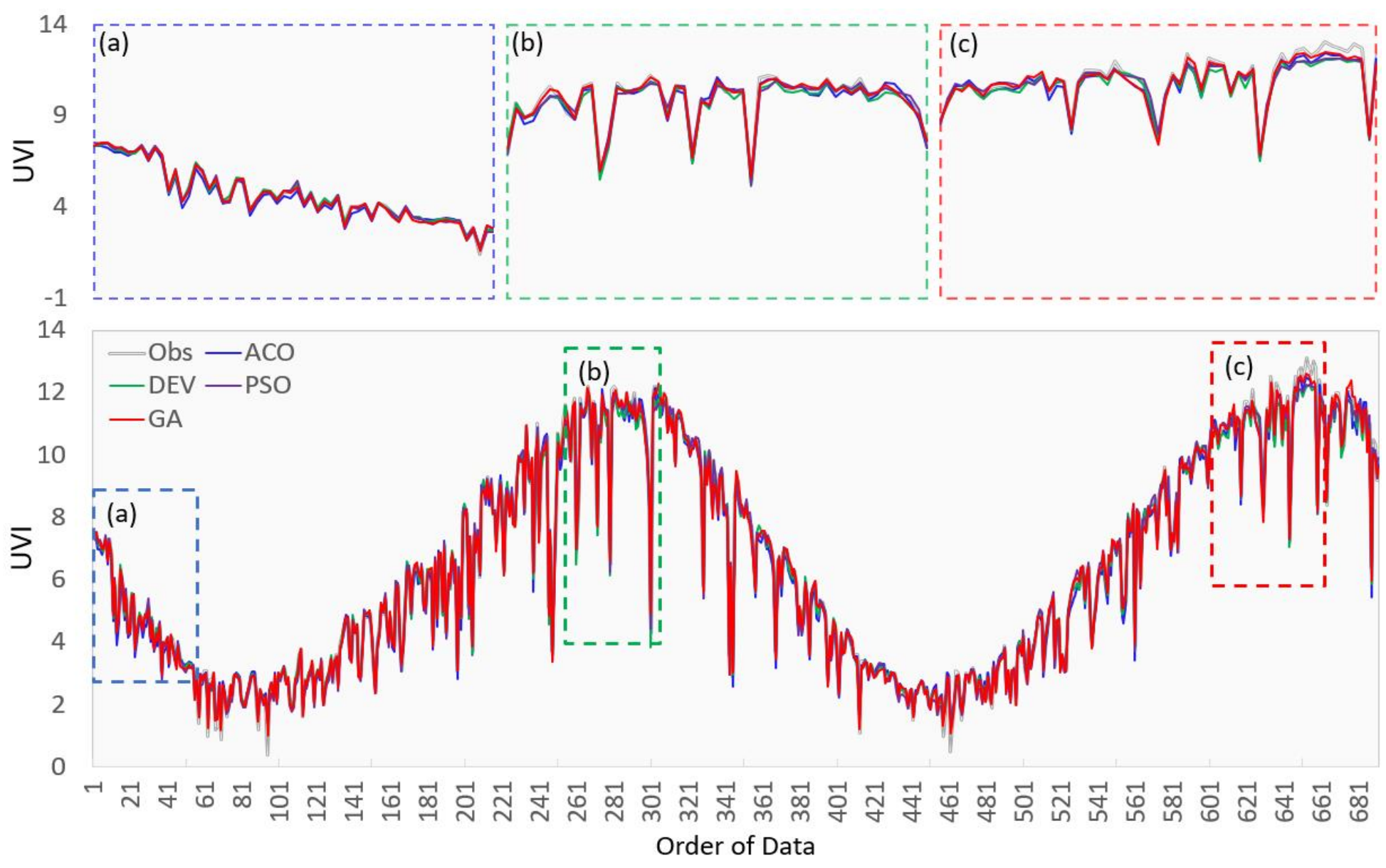

Figure 11 Time series of daily maximum UV index (UVI) for observed UVI and forecasted UVI for the objective model, CEEMDAN-CLSTM using four optimization approaches. 

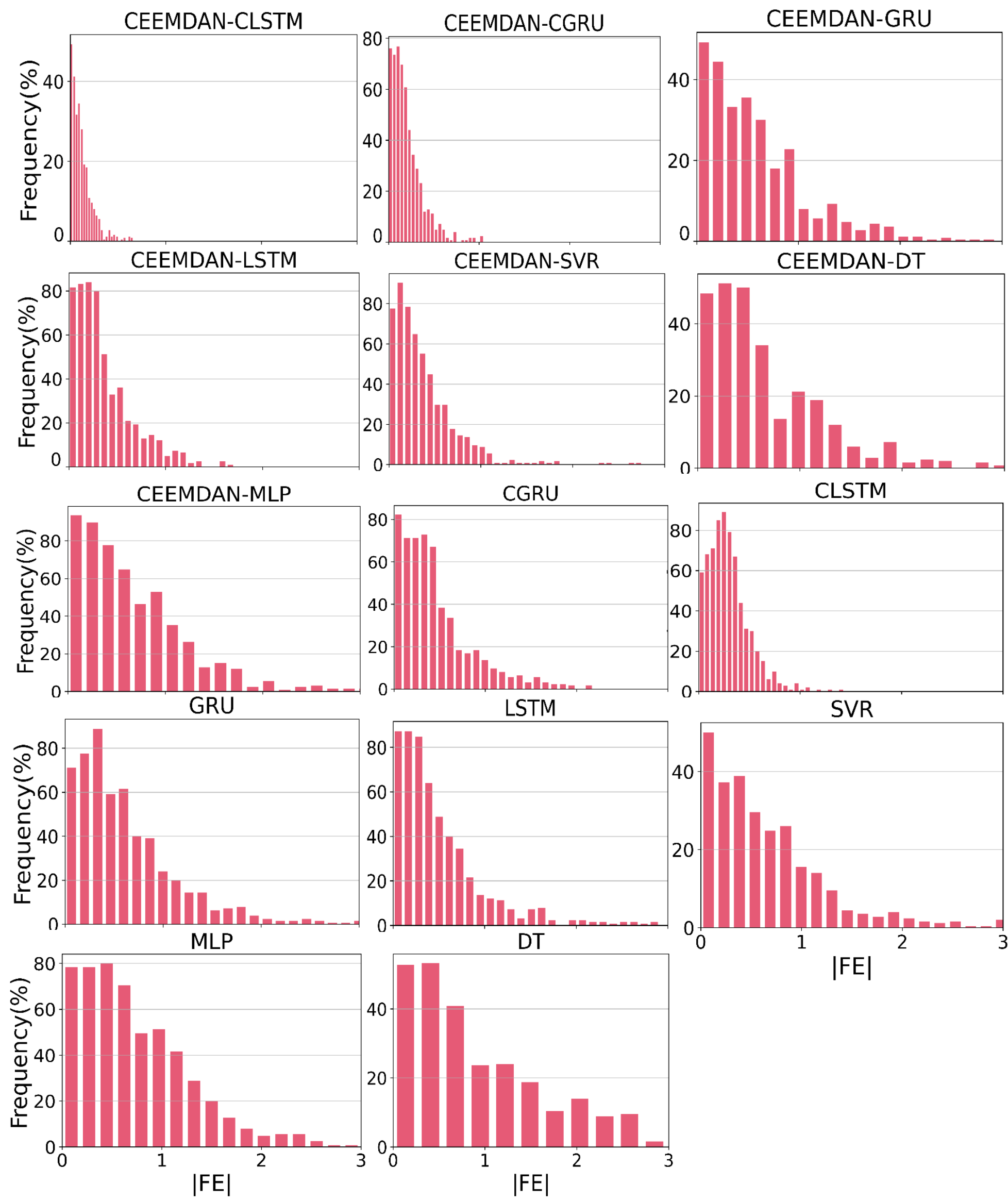

|FE|

Figure 12 Illustration of the frequency of absolute value of estimation errors (|EE|) of the proposed hybrid deep learning CEEMDAN-CNN-LSTM model and comparing models using Genetic Algorithm (GA). 


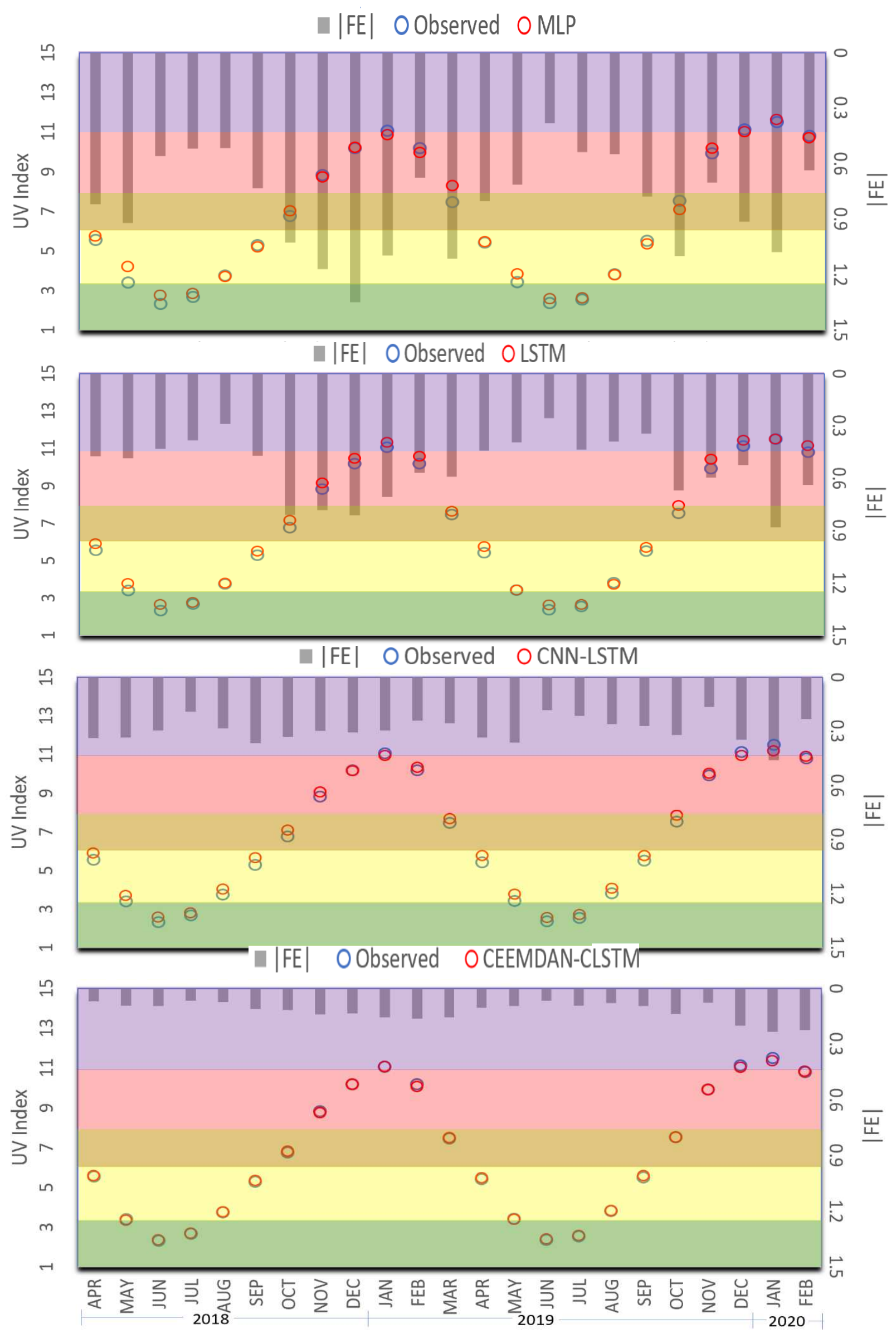

Figure 13 Monthly distribution of observed UVI and forecasted UVI using proposed CEEMDAN-CNN-LSTM model with three benchmark models (i.e., CNN-LSTM, LSTM, MLP) with respective absolute forecasting error $(|\mathrm{FE}|)$ for the testing phase. The color bar indicates the severity of UV values as described in Figure 1. 